\title{
Sorting nexin 3 induces myocardial injury via promoting retromer-dependent nuclear trafficking of STAT3
}

Jing Lu

Sun Yat-sen University

\section{Suowen Xu}

University of Rochester

Yuqing Huo

Augusta University

Yuehuai Hu

Sun Yat-sen University

Juanjian Wang

Sun Yat-sen University

Xiaolei Zhang

Sun Yat-sen University

Duanping Sun

Guangdong Pharmaceutical University

Panxia Wang

Sun Yat-sen University

Zhuo-Ming Li

Sun Yat-sen University

Mengya Liang

First Affiliated Hospital of Sun Yat-sen University

\section{Zhongkai Wu}

First Affiliated Hospital of Sun Yat-sen University

Peiqing Liu ( $\sim$ liupq_sysu@163.com )

Sun Yat-sen University

\section{Article}

Keywords: SNX3, Retromer, STAT3, Nuclear trafficking, Myocardial injury

Posted Date: September 23rd, 2020

DOI: https://doi.org/10.21203/rs.3.rs-77650/v1 
License: (c) (i) This work is licensed under a Creative Commons Attribution 4.0 International License. Read Full License 


\section{Abstract}

Sorting nexins (SNXs), the retromer-associated cargo binding proteins, have emerged as critical regulators of the trafficking of proteins involved in the pathogenesis of diverse diseases. However, studies of SNXs in the development of cardiovascular diseases, especially pathological cardiac hypertrophy, are lacking. Here, we asked whether SNX3, the simplest structured isoform in the SNXs family, may act as a key inducer of myocardial injury. We reported that increased level of SNX3 was observed in failing hearts from human patients and mice. Cardiac-specific Snx3 knockout (Snx3-cKO) in mice significantly protected against isoproterenol (ISO) -induced cardiac injury at 12 weeks. Conversely, cardiac-specific Snx3 transgenic (Snx3-cTg) mice were more susceptible to ISO-induced cardiac injury at 12 weeks, and showed aggravated cardiac injury even heart failure at 24 weeks. Immunoprecipitation-based mass spectrometry (IP-MS), immunofluorescent staining, co-immunoprecipitation and localized surface plasmon resonance (LSPR) were performed to examine the direct interaction of SNX3-retromer with STAT3. STAT3 was discovered as a new interacting partner of SNX3-retromer, and SNX3-retromer served as an essential platform for assembling gp130/JAK2/STAT3 complexes and subsequent phosphorylation of STAT3 by direct combination at early endosomes. SNX3-retromer and STAT3 complexes were transiently imported into the nucleus after hypertrophic stimuli. Moreover, SNX3-retromer promoted importin a3-mediated STAT3 nuclear trafficking and ultimately leading to cardiac injury. Also, pharmacological inhibition of STAT3 reversed SNX3 overexpression-induced myocardial injury in vivo and in vitro. Taken together, our study reveals that SNX3 plays a key role in cardiac function and implicates SNX3 as a potential therapeutic target for cardiac hypertrophy and heart failure.

\section{Introduction}

By responding to various patho-physiological stresses, the heart undergoes a transition from compensatory hypertrophy to decompensatory phase, in which left ventricular remodeling, systolic and diastolic dysfunction and eventual heart failure (HF) occur ${ }^{1-3}$. The abnormal activation of multiple cellular signaling pathways, such as protein kinase $C$ (PKC) -mitogen-activated protein kinase (MAPK), calcineurin-nuclear factor of activated T cells (NFATs) and janus kinase (JAK) -signal transducer and activator of transcription protein (STAT), is the pathological basis for development of heart failure ${ }^{3,4}$.

The physiological functions of signaling proteins are closed linked to their intracellular trafficking and subcellular localization, which is determined by sorting nexins (SNXs)-retromer complex and endosomes ${ }^{5-8}$. SNXs are featured by a highly conserved Phox homology (PX)-domain and comprise 33 members in mammals ${ }^{9}$. Structurally, SNXs directly or indirectly bind to vacuolar protein sorting (VPS) family, VPS26 VPS35 and VPS29, to constitute retromer complex; SNXs also target to endosomal membrane through the PX-domain ${ }^{5,6}$. Functionally, SNXs recruit retromer to early endosomes (EE) or recycling endosomes (RE), and subsequently mediate retrograde transport of cargo proteins via vesicle

budding from EE/RE to trans-Golgi network (TGN), plasma membrane (PM) or cell nucleus ${ }^{5-7}$. SNXs have been reported to regulate diverse disease processes, including Alzheimer's disease, cancer, heart failure 
and arthritis ${ }^{10-13}$. For instance, we previously reported that deficiency of SNX10 prevents inflammation and bone erosion in a mouse model of rheumatoid arthritis through promoting NFATc1 degradation ${ }^{10}$; SNX13 profoundly affects cardiac performance through apoptosis repressor with caspase recruitment domain (ARC) -caspase signaling pathway ${ }^{11}$. Recently, it is found that SNX3 (the simplest structured isoform in the SNXs family)-retromer is required for retrograde recycling of Wntless ${ }^{14}, P C 1$ and PC2 ${ }^{15}$, the cation-independent mannose 6-phosphate receptor (Cl-M6PR) ${ }^{16}$ and iron transporters ${ }^{17}$. However, the role of SNX3 in the pathogenesis of cardiovascular diseases, especially cardiac hypertrophy and heart failure, remains unknown.

Signal transducer and activator of transcription 3 (STAT3), a subtype of STAT family, participates in the pathological process of cardiac hypertrophy and heart failure ${ }^{4,18,19}$. Cardiomyocyte-specific overexpression of STAT3 (Stat3-Tg) in mice causes spontaneous concentric cardiac hypertrophy ${ }^{4}$. In response to stimulation with pro-inflammatory cytokines and growth factors, STAT3 is phosphorylated at tyrosine 705 (Y705) by receptor-associated JAK2, then form homo- or hetero-dimers, and translocate to cell nucleus where they act as transcription activators ${ }^{18-20}$. Importin a3 (also called as Kpna3), a nuclear import factor, is responsible for STAT3 nuclear import independent of tyrosine phosphorylation ${ }^{21,22}$. Upon axons injury exposure, the newly-synthesized STAT3 combined with importin a 5 and dynein (a retrograde molecular motor), and transported back to the cell body ${ }^{23-25}$. STAT3 co-localizes with endocytic vesicles in transit from the cell membrane to the perinuclear region, the perinuclear endosomal compartment to sustain phosphorylated STAT3 (Y705) in nucleus ${ }^{26-29}$. To date, it remains largely unknown whether the retromer-dependent mechanism is involved in the phosphorylation and nuclear trafficking of STAT3.

Here, we demonstrate that the mRNA and protein levels of SNX3 were increased in end-stage failing human hearts and cardiac tissues from isoproterenol (ISO) -induced cardiac injury mouse model. Besides, cardiac-specific Snx3-cKO in mice efficaciously protected against ISO-induced cardiac injury at 12 weeks. Conversely, cardiac-specific Snx3-cTg mice were hypersensitive to ISO-induced cardiac injury at 12 weeks, and resulted in cardiac hypertrophy and dysfunction even heart failure at 24 weeks. Our results reveal the importance of SNX3-retromer complex for assembling gp130/JAK2/STAT3 complexes and subsequent phosphorylation of STAT3 by direct combination at early endosomes. Hypertrophic stimulation-induced nuclear translocation of STAT3 was facilitated by importin a3-mediated SNX3-retromer importing. SNX3retromer promoted STAT3 nuclear trafficking and ultimately leading to abnormal energy metabolism and cardiac injury. Also, pharmacological inhibition of STAT3 by stattic reversed SNX3 overexpressioninduced myocardial injury in vivo and in vitro. Together, our study identifies SNX3 as a potential target for cardiac hypertrophy and heart failure.

\section{Results}

SNX3 expression was up-regulated in human and mouse failing hearts 
To explore the potential role of SNX3 in the development of HF, we analyzed heart samples from 9 patients with HF in end-stage and 3 non-failing healthy controls. The SNX3 mRNA expression was increased in failing human heart tissues compared with that in the normal control tissues as showed by qPCR (Supplementary Figure S1a). The results from immunofluorescence (IF) and western blot analysis suggest that the protein expression of SNX3 was significantly higher in the failing human heart tissues (Fig. 1a and b). Similarly, the mRNA and protein levels of SNX3 were clearly induced in the myocardium of mouse HF model by subcutaneous (s.c.) injection of ISO ( $3 \mathrm{mg} / \mathrm{kg} /$ day, a classic hypertrophic agonist $^{30-32}$ ) for four weeks (Supplementary Figure S1b-d). These results suggest that SNX3 was associated with cardiac hypertrophy and HF in human and mice.

\section{Cardiac-specific Snx3 knockout attenuated ISO-induced myocardial injury in mice}

To examine the function of SNX3 in myocardial injury, cardiac-specific Snx3-cKO mice model was

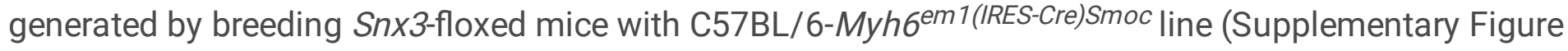
S2a). A cDNA encoding internal ribosome entry site (IRES) and Cre recombinase is inserted into the 3'UTR region of a-myosin heavy chain gene to generate this Myh6-IRES-Cre mouse line ${ }^{33}$. As shown in Fig. 1c and Supplementary Figure. S2f, endogenous SNX3 protein expression was specifically depleted in heart tissues of Snx3-cKO mice, compared with their littermate negative controls (CTL). Snx3-cKO mice showed no obvious cardiac structural or functional defects at basal conditions. To assess whether Snx3 deletion influences cardiac dysfunction under stressed conditions, at 11 weeks, both Snx3-cKO mice and CTL mice were injected with ISO (3 mg/kg/day, s.c.) for one week to establish cardiac injury model. Compared with saline group, ISO treatment significantly induced cardiac hypertrophy, fibrosis and heart dysfunction in $C T L$ mice, evidenced by gross morphologic examination, histological staining, hypertrophic biomarkers (Anp, Bnp and $\beta-M h c)$, heart weight to the tibia length ratio (HW/TL) and echocardiography (Fig. $1 \mathrm{~d}-\mathrm{i}$, Supplementary Figure S3). The results of heart morphology, wheat germ agglutinin (WGA) staining, hematoxylin-eosin (HE) staining, HW/TL ratio, as well as the mRNA levels of $A n p, B n p$ and $\beta-M h c$, demonstrated that ISO treatment induced cardiomyocyte hypertrophy in CTL mice (Fig. 1d-f,

Supplementary Figure S3a and c). Cardiac fibrosis was exhibited by Masson staining and picric sirius red (PSR) staining in CTL + ISO group (Fig. 1g, Supplementary Figure. S3b). The increase of cardiomyocyte size and cardiac fibrosis induced by ISO were significantly reduced in Snx3-cKO mice (Fig. 1d-g, Supplementary Figure. S3a-c). Data of echocardiography, such as ejection fraction (EF), fractional shortening (FS), cardiac output (CO), stroke volume (SV), left ventricular diameter (LVID) and left ventricular volume (LVV), were significantly reduced, while left ventricular mass (LVM), interventricular septum (IVS) and left ventricular posterior wall thickness (LVPW) were increased in ISO group, which was partially recovered by Snx3 knockout (Fig. 1i, Supplementary Figure. S3d-k). These results suggest that knockout of Snx3 significantly alleviated ISO-induced cardiac injury and heart dysfunction in mice.

\section{Cardiac-specific Snx3 transgene leaded to myocardial injury in mice}


To further determine whether SNX3 contributes to myocardial injury, cardiac-specific Snx3 transgenic (Snx3-cTg) mice were generated by crossing Snx3 transgenic mice with B6.FVB-Tg(Myh6-Cre)2182Mds/J mice (Fig. 2a, Supplementary Figure S4). This transgenic strain uses a Cre/loxP approach in which transgenic Cre expression is driven by the mouse Myh6 promoter ${ }^{34}$. Compared with littermate negative controls $(\mathrm{N}-\mathrm{Tg})$, the expression of SNX3 protein is robustly expressed in the heart of Snx3-cTg mice, as indicated by western blot, immunofluorescence (IF) assay and the luciferase activity by live imaging (Fig. 2b, Supplementary Figure S4e-j).

At 12 weeks of age, Snx3-cTg mice showed a mild degree of myocardial hypertrophy, as indicated by the following observations: (1) the larger heart/cardiac size by gross morphological examination, the increased hypertrophic biomarkers and HW/TL ratio, WGA staining and HE staining (Fig. 2c-e, Supplementary Figure S5a and b); (2) deposition of collagen protein by PSR staining (Fig. 2f); (3) echocardiography results (Fig. 2g) showed a decrease in LVID (Fig. 2h), CO (Supplementary Figure S5c), SV (Supplementary Figure S5d) and LVV (Supplementary Figure S5e), as well as an increase in IVS (Fig. 2i), LVM (Supplementary Figure S5f) and LVPW (Supplementary Figure S5g). Overexpression of SNX3 did not apparently affect EF or FS in Snx3-cTg mice at 12 weeks (Fig. 2j, Supplementary Figure S5h).

We also investigated whether overexpression of SNX3 aggravates ISO-induced cardiac hypertrophy in Snx3-cTg mice (12-weeks-old). There were signs of increase of the cardiomyocytes size in both $\mathrm{N}$ - $\mathrm{Tg}$ mice and Snx3-cTg mice; however, Snx3-cTg mice have larger cardiomyocyte size than in N-Tg mice (Fig. 2c-e, Supplementary Figure S5a and b). In addition, ISO-induced cardiac fibrosis was aggravated when SNX3 was overexpressed (Fig. 2f). After ISO infusion, severe heart dysfunction detected by echocardiography was noted in Snx3-cTg mice more than N-Tg mice (Fig. 2g-j, Supplementary Figure S5c-h). These results suggest that Snx3-cTg mice were more susceptible to ISO-induced cardiac injury.

After 24 weeks, Snx3-cTg mice displayed significant hypertrophic growth of cardiomyocytes, increases in $\mathrm{HW} / \mathrm{TL}$ ratios and hypertrophic biomarkers, myocardial fibrosis, compared with $\mathrm{N}-\mathrm{Tg}$ mice (Fig. 2c-f, Supplementary Figure S6a and b). According to the echocardiography (Fig. 2g), Snx3-cTg mice exhibited increases in LVID (Fig. 2h), IVS (Fig. 2i), LVM (Supplementary Figure S6c), and LVV (Supplementary Figure S6d), declines in EF (Fig. 2j), FS (Supplementary Figure S6e), SV (Supplementary Figure S6f) and CO (Supplementary Figure S6g), implying that Snx3-cTg mice spontaneously developed severe heart dysfunction. These results suggest that the cardiac structure was disorganized and systole diastolic performances tend to be worsened with advancing age in Snx3-cTg mice.

\section{SNX3-retromer directly interacted with STAT3 at early endosomes}

The prominent effects of SNX3 on myocardial injury (Fig. 1-2, Supplementary Figure S1-6) prompt us to investigate its underlying mechanisms. Since SNX3 is a key retromer-associated protein ${ }^{5,6}$, we thus examined whether retromer is involved in SNX3-mediated myocardial injury. By immunoprecipitationbased mass spectrometry (IP-MS), we identified STAT3, as a new interacting partner of SNX3, in addition to known interacting proteins, such as retromer proteins VPS26 and VPS35 (Supplementary Figure S7a 
and b). To validate the interaction of SNX3 and STAT3, neonatal rat cardiomyocytes (NRCMs) were infected with Ad-STAT3 (HA-tagged) or Ad-SNX3 (Flag-tagged). Protein interaction was evaluated by IF staining and co-IP assays (Fig. 3a-C). Indeed, IF staining results indicate that HA-labeled STAT3 and retromer proteins (SNX3, VPS26 and VPS35) were located in the same cellular compartments (Fig. 3a). The co-IP results further suggest that SNX3-retromer interacted with STAT3 in NRCMs and mouse heart tissues (Fig. 3b and c, Supplementary Figure S7c-e). Moreover, according to LSPR results, recombinant STAT3 protein (117-770aa) directly interacted with SNX3, VPS35 and VPS26 with a binding constant at 7.10e-7 M, 1.58e-8 M, 1.30e-8 M (KD values), respectively (Supplementary Figure S7f-I). The interaction between truncated STAT3 protein (576-678aa) and SNX3-retromer were shown in Fig. 3d with a binding constant at 6.11e-8 M, 5.06e-8 M, 1.01e-7 M (KD values) in cell-free system.

Besides, NRCMs were precipitated by anti-early endosome antigen 1 (EEA1), Rab5 and clathrin heavy chain (CHC) (markers of early endosome), SNX3 and STAT3 were found in the precipitation of early endosome by co-IP assays. (Fig. 3e, Supplementary Figure S7m and $n$ ). The early endosome fraction was purified from NRCMs using density gradient centrifugation, and detected by western blot analysis (Fig. 3f). We observed that both STAT3 and SNX3 mainly associated with early endosomes in NRCMs, though some STAT3 positive staining was present in late endosomes and lysosomes, as suggested by STAT3 combining with the endosomal compartments markers (EEA1, Rab5, Rab7, CHC and Lamp-2) (Fig. 3b, C, e, f, Supplementary Figure S7m-p). These results imply that STAT3 directly interacted with SNX3-retromer at early endosome in cardiomyocytes.

\section{SNX3-retromer served as a platform for STAT3 activation}

It is well-established that JAK2/STAT3 pathway is involved in the pathogenesis of cardiac hypertrophy and heart failure ${ }^{4}$. To investigate whether SNX3 cooperates with STAT3 to mediate cardiac hypertrophy, we established three classical cardiac hypertrophic models (induced by ISO, angiotensin $\otimes$ (Ang $\nabla$ ) and phenylephrine (PE), respectively) in NRCMs. According to the increase of cell surface area and increased mRNA expression of hypertrophic markers, three models of cardiomyocyte hypertrophy were successfully established (Fig. 4a, b). The phosphorylation levels of JAK2 (Y1007 and Y1008) and STAT3 (Y705), and the protein expression of SNX3 were enhanced in these hypertrophic models, whereas the protein levels of gp130 and STAT3 remained unchanged (Fig. 4c, Supplementary Figure S8a). The homo-/hetero dimerization of STAT3 and STAT1 was also increased by treatment with hypertrophy stimuli (Supplementary Figure S8b).

Given that SNX3 is localized in retromer-containing endosomes ${ }^{6}$, we investigated whether SNX3-retromer is involved in JAK2/STAT3 signaling. The results of co-IP and IF assay showed that ISO increased the association of STAT3 with SNX3, JAK2 and gp130, whereas knockdown of SNX3 decreased these interactions (Fig. 4d-f). Consistently, knockdown of SNX3 significantly inhibited ISO-induced phosphorylation and dimerization of STAT3 (Fig. $4 \mathrm{~g}$ and $\mathrm{h}$ ), whereas overexpression of SNX3 by infecting with Ad-SNX3 had the opposite effects (Supplementary Figure S8c-e). Collectively, these results suggest 
that SNX3-retromer serves as a platform for the assembly of gp130/JAK2/STAT3 complexes and the subsequent phosphorylation of STAT3 in ISO-induced cardiac hypertrophy.

\section{SNX3-retromer promoted the nuclear localization of STAT3}

After treatment with stimuli of cardiac hypertrophy, cytoplasmic STAT3 shuttles to the nucleus, where STAT3 orchastreates hypertrophy-related target gene expression (such as Anf, c-fos, c-myc, aGT) ) $^{4,35,36}$. Given that SNX3-retromer complex plays a vital role in sorting cargoes from endosomes to the intracellular trafficking ${ }^{6}$, we examined the effects of SNX3-retromer on the subcellular distribution of STAT3 in the process of cardiac hypertrophy. NRCMs were treated with ISO, Ang $\triangle$ or PE, or were infected with Ad-SNX3 or Ad-sh-SNX3 before ISO treatment. The nuclear and cytoplasmic proteins were extracted from NRCMs, and were detected by western blot analysis. The results showed that transient nuclear import of STAT3, SNX3, VPS35 and VPS26, after treatment with hypertrophic stimuli for $1 \mathrm{~h}$ (Supplementary Figure S9a). By contrast, SNX3 silencing reversed ISO-induced nuclear import of STAT3 using IF and western blot analysis (Fig. 5a and b, Supplementary Figure S9b). Similarly, the ISO-induced increase of transcriptional activity and target gene expression of STAT3 was inhibited by knockdown of SNX3 using luciferase reporter assay and qPCR (Fig. $5 \mathrm{c}$ and d). The above findings revealed that nuclear translocation of STAT3 was depends on SNX3-retromer complex.

Previous studies have shown that importin a3, a nuclear importing factor, is responsible for STAT3 nuclear import induced by hormones or intracellular tyrosine kinases ${ }^{21,22}$. The endogenous importin a3 was knocked down using the appropriate shRNA in NRCMs (Supplementary Figure S9c and d). By IF staining, we observed that Ad-SNX3 treatment significantly increased ISO-induced interaction of importin a3 and STAT3-SNX3-retromer complexes (Fig. 5e). However, importin a3 knockdown attenuated Ad-SNX3triggered the nuclear importing of STAT3 and SNX3-retromer (Fig. $5 f$ and g, Supplementary Figure S9e), suggesting that SNX3-retromer promoted the importin a3-mediated nuclear localization of STAT3 in cardiomyocytes.

\section{Involvement of STAT3 in SNX3-mediated cardiomyocyte injury}

We then asked whether SNX3 induces cardiomyocyte injury through STAT3 activation. To this end, NRCMs were infected with Ad-sh-SNX3 before ISO treatment, or were infected with Ad-SNX3 with or without stattic (a specific inhibitor of STAT3) ${ }^{28}$. We observed that knockdown of SNX3 significantly suppressed the hypertrophic growth of cardiomyocytes induced by ISO (Fig. 6a, b). Conversely, Ad-SNX3 led to increased hypertrophic responses, which was partly inhibited by stattic, implying that STAT3 was involved in SNX3-mediated cardiomyocyte hypertrophy (Fig. 6a, b). Given that the progression of cardiac hypertrophy is characterized by disturbed mitochondrial energy metabolism ${ }^{1}$, we then measured the rate of oxygen consumption (OCR) with the high-throughput analyzer Seahorse XF96. The results from Fig. 6c showed that OCR was significantly increased in ISO or Ad-SNX3-stimulated cardiomyocytes, both of 
which were inhibited by Ad-sh-SNX3 or stattic. Interestingly, Ad-SNX3 increased basal respiration, ATP production, proton leak, spare respiratory capacity, maximum respiration and non-mitochondrial oxygen consumption, which was partly inhibited by stattic (Fig. 6c, Supplementary Figure S10a-f). Similarly, Adsh-SNX3 decreased the ISO-induced OCR indices (Fig. 6c, Supplementary Figure S10g-I). These data pinpointed a critical role of STAT3 and SNX3 in ISO-induced cardiac hypertrophy and abnormal mitochondrial energy metabolism.

To further confirm the role of STAT3 in SNX3-induced cardiac injury in vivo, Snx3-cTg mice were treated with stattic ( $40 \mathrm{mg} / \mathrm{kg} / \mathrm{d}$, i.p.) or an equal volume of vehicle for 2 weeks. The phosphorylation level of STAT3 at the tyrosine 705 (pY705) was significantly inhibited by stattic, suggesting that the activation STAT3 was inhibited by stattic (Fig. 6e, f). After treatment with stattic, $N$-Tg mice showed no noticeable changes in cardiomyocytes (Fig. 6g-j, Supplementary Figure S11a-l). However, the degree of cardiac hypertrophy (determined by gross observation of heart morphology, hypertrophic biomarkers, HW/TL ratio, WGA staining and HE staining) of Snx3-cTg mice was significantly alleviated in stattic treatment group compared with that in vehicle-treated group (Fig. 6g, Supplementary Figure S11a, b, d, e). Besides, Snx3-cTg mice showed increased cardiomyocyte interstitial fibrosis (determined by PSR staining and Masson staining), while that effect was widely suppressed upon stattic treatment (Fig. 6h, Supplementary Figure S11c). The echocardiography data showed that Snx3-cTg reduced EF, FS, CO, LVV, LVID and SV, increased LVPW, LVM and IVS, which could be antagonized by stattic in different degree (Fig. 6j, Supplementary Figure S11f-m).

These results suggest that STAT3 was involved SNX3 overexpression-induced myocardial injury in cardiomyocytes and in Snx3-cTg mice.

\section{Discussion}

SNX3 plays a crucial role in the pathogenesis of diverse diseases, including Parkinson's disease ${ }^{17}$, Alzheimer disease ${ }^{37}$, autosomal dominant polycystic kidney disease ${ }^{15}$. However, it remains unknown whether SNX3 plays a role in cardiac diseases. In this study, we observed that SNX3 expression was increased in end-stage failing human hearts and cardiac tissues from mouse model of ISO-induced cardiac injury. By using gain- and loss-of-function experiments in vivo, we revealed a novel role of SNX3 in the development of cardiac hypertrophy and heart failure. This was evidenced by the fact that cardiacspecific Snx3-cKO protected mice against ISO-induced cardiac injury, conversely, Snx3-cTg mice were hypersensitive to ISO-induced cardiac hypertrophy and aggravated cardiac injury with advancing age.

It has been reported that SNXs family affect a wide range of biological processes by regulating the intracellular trafficking of diverse signaling proteins ${ }^{10-13,38}$. For instance, Deficiency of SNX10 prevents inflammation and bone erosion in rheumatoid mouse arthritis through promoting NFATc1 degradation ${ }^{10}$; SNX13 profoundly affects cardiac performance through the SNX13-PXA-ARC-caspase signaling 
pathway ${ }^{11}$; Deletion of SNX27 reverses epithelial-mesenchymal-transition in highly aggressive breast cancer cells ${ }^{13}$; SNX27 serves as an essential adaptor protein, mediates beta 2ARs to the retromer tubule and endosome-to-plasma membrane trafficking ${ }^{38}$. However, the effect of SNX3 on the intracellular transportation of cargo proteins, which is tightly associated with cardiovascular diseases, has not been evaluated yet. Here, we identify the specific role of SNX3-STAT3 interaction in cardiomyocytes. SNX3 was required for STAT3 activation, inhibition of STAT3 could reduce the detrimental role of overexpressed SNX3 in cardiomyocytes and Snx3-cTg mice.

It was previously believed that retromer (VPS26-VPS35-VPS29 heterotrimers) act as the cargo-sorting complex ${ }^{39,40}$. Recent studies have shown that SNX proteins are indispensable for cargo recognition, select and binding ${ }^{6,41}$. A T-shaped architecture of SNX3-retromer was identified, and this complex comprised SNX3, VPS26 and VPS35 ${ }^{6}$. SNX3-retromer complex play the central role in recycling various proteins (such as Wnt, Wntless, transferrin receptor, Fet3-Ftr1 and $\beta-A R$ ) from the endosomes to TGN or $\mathrm{PM}^{14,17,37,42-46}$. SNX3-retromer mediate Wntless sorting and Wnt secretion ${ }^{14,42-44}$. SNX3 regulates the recycling of transferrin receptor and iron assimilation ${ }^{37}$. SNX3-retromer mediate retrograde recycling of iron transporters in S. cerevisiae and C. elegans models of Parkinson's disease ${ }^{17,37}$. In iron-starved cells, Fet3-Ftr1 is sorted by SNX3/Grd19 and retromer into a recycling pathway that delivers it back to the $\mathrm{PM}^{45,46}$. In this study, we identified STAT3 as a new interacting partner of SNX3-retromer complex mainly in early endosomes in cardiomyocytes.

STAT3 activity is tightly regulated for proper physiological processes, and its aberrant and persistent activation will result in pathological cardiac hypertrophy ${ }^{4,18}$. Over-activation of STAT3 could be mainly attributed to either the stimulation of STAT3 activators (such as ISO and IL-6), or the aberrantly activated upstream tyrosine kinases (such as JAK2 and EGFR) ${ }^{18-20}$. The present work suggest that SNX3-retromer acts as an essential platform for the assembly of gp130/JAK2/STAT3 complexes induced by ISO, and subsequent phosphorylation of STAT3 by direct combining at early endosomes.

To date, the main research of SNXs-mediated intracellular transportation is focused on the subcellular trafficking from the endosomes to $\mathrm{TGN}$ or $\mathrm{PM}^{6}$. It is rarely reported that the nuclear transport of intracellular proteins mediated by SNXs, except that SNX11 is required for the translocation of factor II receptor-like 1 ( $F 2$ rl1) from the PM to the cell nucleus in retinal ganglion cells ${ }^{7}$. We report that SNX3retromer complex was transiently imported into the nucleus after hypertrophic stimuli, and promoted importin a3-dependent nuclear translocation of STAT3 in the cardiac hypertrophy model.

According to our work, it is plausible that SNX3-retromer promotes both STAT3 activation and nuclear translocation. The detailed mechanism whereby the complex is recruited to membrane receptor to be activated and subsequently directed to nuclear after activation is not clear yet. There may be another protein or posttranslational modification determines the fate of the complex for intracellular trafficking. 
In conclusion, the present study demonstrates that SNX3-retromer plays a critical role in cardiac function and suggests that SNX3 could be exploited as a potentially new therapeutic target for cardiac hypertrophy and heart failure.

\section{Methods}

\section{Human heart samples}

The study conforms to the principles that govern the use of human tissues outlined in the Declaration of Helsinki. Approval was obtained from the human ethics committee of First Affiliated Hospital of Sun Yatsen University. All patients or the family of prospective heart donors gave written informed consent prior to participation. Failing human heart samples were collected from 9 patients undergoing heart transplantation because of end-stage heart failure (table S1). Three non-failing control heart samples were obtained from prospective multi-organ donors, which did not exhibit cardiovascular pathology but were unable to be transplanted due to technical reasons. Tissue samples were collected at the time of explantation and rapidly frozen in liquid nitrogen or fixed with $4 \%$ paraformaldehyde.

\section{Animal Studies}

Snx3-floxed mice were constructed in the Shanghai Model Organisms Center by CRISPR/Cas 9 technology. Briefly, the donor vector containing four guide RNAs and Cas9 mRNA targeting Snx3 introns 2 and 3 was microinjected into C57BL/ 6 mouse fertilized eggs. The positive founder mice were backcrossed with wild-type C57BL/ 6 mice to obtain heterozygous $S n \times 3^{\text {flox/+ }}$ mice with germ line transmission. Snx $3^{\text {flox/+ }}$ mice were self-crossed to generate homozygous $\operatorname{Sn} x 3^{\text {flox/flox }}$ mice. Snx $3^{\text {flox/flox }}$

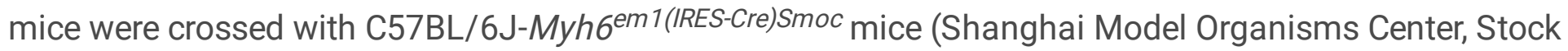
No: NM-KI-00083. MGI ID: 97255) to generate Myh6-Cre+; Snx $3^{\text {flox/flox }}$ mice, ie. cardiac-specific Snx3-cKO mice.

Snx3 transgenic mice were constructed in the Shanghai Model Organisms Center using standard methods. Briefly, the Piggybac vector harboring mouse SNX3 CDNA was microinjected into the fertilized egg of C57BL/ 6 mouse, and the transgenic founder mice were obtained. 3 generations of backcross between each Snx3 transgenic mice and wild-type (C57BL/6) mice were adopted to breed two independent Snx3 transgenic lines. The two transgenic lines were respectively crossed with B6.FVBTg(Myh6-Cre)2182Mds/J mice (Jackson Laboratory (Bar Harbor, ME), Stock No: 011038. MGI ID: 2386742.) to generate cardiac-specific overexpressed SnX3 (Snx3-cTg) mice.

The mice were genotyped by PCR and further confirmed by western blot analysis (table S2-S4). All animal protocols were conducted under the institutional guidelines of Animal Care and Use Committee, and were approved by the Research Ethics Committee, Sun Yat-sen University. Experimental animals were housed, bred, and maintained in the specific pathogen-free (SPF) facility of the Experimental Animal Center of Sun Yat-sen University. As we previously described ${ }^{30}$, the transthoracic 2D-guided M-mode 
echocardiography (such as heart function and global cardiac volumes) was assessed using by a Technos MPX ultrasound system (ESAOTE, SPAESAOTE SpA, Italy) equipped with an 40-MHz scan probe. The Vevo 2100 imaging software was used for measurements and calculations. Then, the treated mice were sacrificed, and hearts were rapidly sampled for further experiments.

\section{Bioluminescence Imaging ${ }^{47}$}

XenoLight $^{\mathrm{TM}}$ D-luciferin potassisum salt (PerkinElmer, $\mathrm{P} / \mathrm{N}$ 122799) was diluted in sterile PBS to 15 $\mathrm{mg} / \mathrm{mL}$, and was respectively injected into the luciferase-labeled Snx3-cTg mice or $N$-Tg mice (150 $\mathrm{mg} / \mathrm{kg}$ ). A few minutes after injection, mice were anesthetized using $2 \%$ isoflurane inhalation (with a 2 $\mathrm{L} /$ min oxygen flow rate), and were placed inside the camera box of the IVIS Lumina XR small animal optical imaging system (PerkinElmer). The sequential images of the mice were run every two minutes. Lumazone Version 2.0 software was used to analyze the intensity of the fluorescence (intensity/second) in mice.

\section{Histological Analysis}

Myocardial tissue samples were fixed in 10\% paraformaldehyde and embedded in paraffin for sectioning. Sections were stained with wheat germ agglutinin (WGA), hematoxylin-eosin (HE), Masson's and picric sirius red (PSR) for histopathological examination under a light microscope.

For immunofluorescent (IF), paraffin sections of myocardial samples were treated with primary anti-SNX3 (Proteintech, \#10772-1-AP) or anti-p-STAT3 (Y705, Cell Signaling Technology, CST, Beverly, MA, USA, \#9145) overnight at $4^{\circ} \mathrm{C}$. Then, the samples were incubated with CoraLite488/594-conjugated anti-rabbit IgG (diluted 1:200, Proteintech, \#SA00013-6 or \#SA00013-8), were counter-stained with 4',6-diamidino-2phenylindole (DAPI, CST, \#4083). Fluorescence was captured by EVOS FL Auto (Life Technologies, Bothell, WA, USA).

\section{Plasmid, recombinant adenoviral vectors and recombinant protein}

Vps26(NM_001007740.1), Vps35(NM_001105718.2), Snx3 (NM_001044283.1) and Stat3 (NM_012747.2) were constructed by ligating respective full-length cDNA into pcDNA3.1 (+) with Flag or HA-tag. Similarly, short hairpin (sh) RNA targeting the rats importin a3 gene (also called as Kpna3, NM_001014792) (sh-importin a3), the rats Snx3 gene (sh-SNX3) and non-targeting control shRNA (sh-NC) sequences (table S5 and S6) were respectively subcloned into pcDNA3.1 (+) with Flag-tag.

Recombinant adenoviral vectors expressing rats $S n \times 3$ cDNA (Ad-SNX3, with Flag-tag), rats STAT3 cDNA (Ad-STAT3, with HA-tag), sh-SNX3 sequence (Ad-sh-SNX3, with Flag-tag) and control vectors were generated by standard procedures. Briefly, the pAdTrack plasmids containing corresponding genes were linearized by using restriction endonuclease $P m e \Downarrow$, and were transformed into Escherichia coli strain BJ5183 cells carrying the Ad-Easy-1 plasmid. The successful recombinant plasmids were digested with restriction endonuclease Pac®, and were transfected into HEK293A cells to generate Ad-SNX3, Ad-STAT3 
or Ad-sh-SNX3. The vectors were purified by plaque, cultured in a large scale, and purified by CsCl stepand isopycnic-gradient centrifugation.

GST-STAT3, GST-SNX3, GST-VPS35 and GST-VPS26 protein were respectively expressed in E. coli BL21 (DE3) maintained in luria-bertani (LB) medium (including $50 \mathrm{ug} / \mathrm{mL}$ ampicillin) at $37^{\circ} \mathrm{C}$. By the addition of isopropyl- $\beta$-D-thiogalactopyranoside (IPTG, $0.5 \mathrm{mmol} / \mathrm{L}$ ), protein expression was induced at $16^{\circ} \mathrm{C}$ overnight to an OD600 value of 0.8 . Cells were re-suspended in $20 \mathrm{mmol} / \mathrm{L}$ Tris $+200 \mathrm{mmol} / \mathrm{L} \mathrm{NaCl}+1$ $\mathrm{mmol} / \mathrm{L}$ PMSF, were broken using ultrasonic instrument in ice, and were collected supernatant after centrifugation $\left(1,2000 \mathrm{rpm}\right.$ for $3 \mathrm{~h}$ at $\left.4^{\circ} \mathrm{C}\right)$. These recombinant protein were purified by GST-Sefinose Gravity Column, were enzyme digested at room temperature overnight. All purified protein were dissolved in sterile phosphate-buffered saline (PBS), were measured their concentration and stored at $-80^{\circ} \mathrm{C}$.

\section{Localized surface plasmon resonance (LSPR) Assays ${ }^{48}$}

To examine the direct interaction between STAT3 and SNX3-retromer, LSPR assays were conducted on an OpenSPR system (Nicoya Lifesciences, Waterloo, Canada). Recombinant proteins STAT3 (117-770aa, 576-678aa), SNX3 and VPS35 served as the ligand and were respectively immobilized on a gold nanoparticle sensor chip via capture-coupling. Subsequently, the recombinant protein SNX3, VPS35 and VPS26 at different concentrations were sequentially injected into the chamber in running buffer (filtered PBS) with a constant flow rate of $20 \mu \mathrm{L} / \mathrm{min}$, and were passed over the sensor (about $5 \mathrm{~min}$ ) for the association of two protein. Following each recombinant protein injection (all concentrations were performed in triplicate), the chip was completely dissociated with the complex and regenerated by injecting hydrochloric acid ( $\mathrm{pH} 2.0)$. As recommended by the manufacturer, the results were analyzed by Trace Drawer software (Ridgeview Instruments AB). The kinetic parameters, including the association constant (ka), dissociation constant $(k d)$ and affinity $(K D, K D=k d / k a)$, were calculated by a simple 1: 1 diffusion corrected model, which adjusted to the wavelength shifts consisting with the varied concentration of protein.

\section{Primary culture of neonatal rat cardiomyocytes (NRCMs)}

As reported before ${ }^{30}$, NRCMs were isolated from the hearts of one to three-day-old SD rats. Cardiomyocytes were plated into 6-well microplates (Corning, USA) comprising Dulbecco's modified Eagle's medium (DMEM, Gibco, USA) supplemented with $10 \%$ newborn calf serum (NBCS) and 5bromodeoxyuridine (Sigma, \#B5002, $0.1 \mathrm{mmol} / \mathrm{L}$ ), at a density of $1 \times 10^{6}$ cells/well.

\section{Plasmid Transfection and virus infections ${ }^{30}$}

NRCMs were transfected with sh-SNX3 plasmid together with lipofectamine 3000 reagent (Invitrogen, USA) in OptiMEM medium as per the manufacturer's instructions. The medium was changed to DMEM complete medium after eight to seventy two hours of transfection. NRCMs were infected with recombinant adenoviruses (including Flag-tagged SNX3, Flag-tagged sh-SNX3 or HA-tagged STAT3) at a 
multiplicity of infection (MOI) of 20 particles per 5 cells. Western blot and/or Quantitative RT-PCR were performed to confirm the efficiency of overexpression or depletion.

\section{Mitochondrial respiration assay by Seahorse XF96 ${ }^{49}$}

The prepared cardiomyocytes were seeded in a Seahorse XF96 polystyrene cell culture microplate (Seahorse Bioscience, North Billerica, USA), and incubated in a cell culture incubator at $37^{\circ} \mathrm{C}$ overnight. The next day, the medium was changed to XF base Medium (Seahorse Bioscience) containing 10 $\mathrm{mmol} / \mathrm{L}$ glucose (Sigma), $1 \mathrm{mmol} / \mathrm{L}$ pyruvate (Sigma), and $2 \mathrm{mmol} / \mathrm{L}$ glutamine (Sigma) for the detection of basal respiration. Some reagents were serially injected to detect ATP production (mitochondria respiration), maximal respiration and non-mitochondrial respiration, including oligomycin $(2 \mu \mathrm{mol} / \mathrm{L}$, inhibits ATP synthase (complex $\nabla)$, FCCP $(1 \mu \mathrm{mol} / \mathrm{L}$, uncouples oxygen consumption from ATP production), and a mix of rotenone $(0.5 \mu \mathrm{mol} / \mathrm{L}$, inhibits complexes $\nabla)$ and antimycin $A(0.5 \mu \mathrm{mol} / \mathrm{L}$, inhibits complexes $\mathbb{\nabla}$ ). Proton leak and spare respiratory capacity were calculated by these parameters using a high-throughput $\mathrm{XF}^{\mathrm{e}} 96$ extracellular flux analyzer (Seahorse Bioscience).

\section{Measurement of the cell surface area ${ }^{30,50,51}$}

NRCMs were seeded in 24-well microplates, fixed with paraformaldehyde (4\%, Beyotime, \#P0099) for 10 min at room temperature. After permeabilizing with Triton X-100 (0.3\%, Beyotime, \#P0096) and blocking with goat serum (Beyotime, \#C0265), the cells were incubated with primary antibody myosin light chain 2 (MLC2, Proteintech, \#10906-1-AP) overnight at $4^{\circ} \mathrm{C}$, treated with secondary antibody anti-alexa fluor 488 (Proteintech, \#SA00013-6, for 2h) and rhodamine-phalloidin (0.1\%, Invitrogen \#R415, for $30 \mathrm{~min}$ ) at room temperature. After washing with filtered PBS, NRCMs were mounted by using DAPI (CST, \#4083). The images were taken using the High Content Screening System (Thermo Fisher Scientific, USA), and the cell surface area from randomly selected fields (fifty for each group) was analyzed by the built-in image analysis software.

\section{Isolation of early endosomes by continuous density gradient centrifugation ${ }^{52,53}$}

The nuclear and cytoplasmic protein was successively extracted from NRCMs using a kit (SC-003, Inventbiotech, MN, USA). The postnuclear fraction was suspended in buffer, and was used to a continuous sucrose density gradient. After centrifugation $\left(210,000 \mathrm{~g}\right.$ for $3 \mathrm{~h}$ at $\left.4^{\circ} \mathrm{C}\right)$, a milky band should be visible at each interface. 24 consecutive fractions were collected from each interface into tubes, and were subjected to western blot analysis for detection of protein EEA1, which is an early endosomal marker.

\section{Low temperature SDS-PAGE, Western blot and co-immunoprecipitation (co-IP) analysis}

Low-temperature SDS-PAGE was conducted to investigate the homodimers (STAT3/STAT3) or heterodimer (STAT3/STAT1). In short, total protein from NRCMs were incubated in loading buffer (without 2-mercaptoethanol) for $5 \mathrm{~min}$ at $37^{\circ} \mathrm{C}$, were separated by $8 \%$ SDS-PAGE for $4-5 \mathrm{~h}$ at a constant current of 
$40 \mathrm{~mA}$, and were transferred to a PVDF membranes (Millipore). The whole process of the experiment must be made under the low temperature. After blocking with 10\% blocking buffer (Beyotime, \#P0023B), the membranes were incubated with individual antibodies at $4^{\circ} \mathrm{C}$ overnight ${ }^{54}$.

Western blot analysis was performed as previously reported ${ }^{30,50}$. Immunoblots were labelled with the following primary antibodies: primary antibodies against p-JAK2 (Y1007 and Y1008, rabbit, diluted 1:500, \#3776), JAK2 (rabbit, diluted 1:1,000, \#3230), gp130 (rabbit, diluted 1:1,000, \#3732), p-STAT3 (Y705, rabbit, diluted 1:1,000, \#9145), STAT3 (rabbit, diluted 1:2,000, \#9139) were bought form CST. Primary antibodies against clathrin heavy chain (CHC, rabbit, diluted 1:1,000), EEA1 (rabbit, diluted 1:1,000) and Rab5 (rabbit, diluted 1:1,000) were purchased from CST (endosomal marker antibody sampler kit, \#12666). SNX3 (rabbit, diluted 1:1,000, \#10772-1-AP), VPS26 (rabbit, diluted 1:800, \#15915-1-AP) and VPS35 (rabbit, diluted 1:800,\#10236-1-AP) were products of Proteintech. Primary antibodies against importin a3 (rabbit, diluted 1:1,000, \#19783), HA (rabbit, diluted 1:5,000, \#H6908) and a-tubulin (mouse, diluted 1:5,000, \#T8203) were purchased from Sigma-Aldrich. Anti-Flag (mouse, diluted 1:5,000, \#PM185), anti-Lamin B1 (rabbit, diluted 1:1,000, \# PM064) were purchased from MBL. The enzyme horseradish peroxidase (HRP) -conjugated secondary antibodies (CST, \#7074 and \#7076) were applied to chemiluminescence detection and the protein band intensities were quantified by LabWorks software (Bio-Rad, USA).

For co-IP, anti-EEA1 (rabbit, diluted 1:50), anti-CHC (rabbit, diluted 1:50), anti-Rab5 (rabbit, diluted 1:20) were purchased from CST (endosomal marker antibody sampler kit, \#12666) Anti-VPS26 (rabbit, diluted 1:20, \#15915-1-AP) and anti-VPS35 (rabbit, diluted 1:20, \#10236-1-AP) were products of Proteintech. AntiFlag (rabbit, diluted 1:50, \#PM185) was purchased from MBL. Anti-HA (rabbit, diluted 1:50, \#H6908) was purchased from Sigma-Aldrich. The rabbit normal IgG (\#3900) and mouse normal IgG (\#53484) were purchased from CST. NRCMs were harvested with IP lysis buffer (Beyotime, \# P0013) supplemented with protease and phosphatase inhibitor cocktails (Bimake, \#B14012 and \#B15002). After clarification by centrifugation, $400-600 \mu \mathrm{g}$ of total protein cell lysate were incubated with the indicated primary antibodies overnight at $4^{\circ} \mathrm{C}$, and were incubated with protein G-agarose beads (Pierce, Rockford, IL, USA) at $4^{\circ} \mathrm{C}$ for $4 \mathrm{~h}$. Normal $\lg \mathrm{G}$ was served as an control. The immunoprecipitated proteins were detected by western blot or mass spectrum analysis.

\section{Immunofluorescence (IF) assay}

NRCMs were cultured in chamber slides (ThermoFisher Scientific). After treatment, cells were washed with filtered PBS for 3 times, fixed with $4 \%$ paraformaldehyde for 10 min, permeabilized with $0.3 \%$ Triton $\mathrm{X}-100$ for $5 \mathrm{~min}$ and followed by blocking with goat serum for $1 \mathrm{~h}$ at room temperature. The cells were further treated with the following primary antibodies overnight at $4^{\circ} \mathrm{C}$ : STAT3 (CST, \#9139), SNX3 (Proteintech, \#10772-1-AP), VPS35 (Proteintech, \#10236-1-AP), VPS26 (Proteintech, \#15915-1-AP), EEA1 (CST, \#12666), Rab5 (CST, \#12666), Rab7 (CST, \#12666), CHC (CST, \#12666), Lamp-2 (Proteintech, \#66301-1-lg), gp130 (CST, \#3732), JAK2 (CST, \#3230) and importin a3 (Sigma-Aldrich, \#19783). Fluorescence emitted by fluorescence-conjugated secondary antibodies (Proteintech, \#SA00013-6 and 
\#SA00013-8) at room temperature for $2 \mathrm{~h}$. The slides were mounted with DAPI (CST, \#4083) and were observed by a confocal microscope (Zeiss, Germany) or EVOS FL Auto (Life Technologies).

\section{Total RNA isolation, cDNA Synthesis and real-time polymerase chain reaction (qPCR)}

Total RNA was extracted from snap-frozen cardiac tissues or NRCMs by using Trizol reagent (Invitrogen, \#15596026), and its concentration was measured with a Nanodrop 2000 (Thermo Fisher Scientific, USA). The RNA extract (1000 ng) was reversely transcribed to first strand cDNA using the One-step Reverse Transcription (RT) Kit (Thermo Fisher Scientific, USA). Quantitative SYBR Green-based PCR (TOYOBO, Janpan) was conducted on the iCycler iQ system (Bio-Rad, USA). GAPDH was used as reference gene. All PCR assays were performed in triplicate. Data were analyzed using the $2^{-\triangle \Delta C T}$ method. The oligonucleotide sequences are synthesized by Sangon (Shanghai, China), and listed in table S7.

\section{Dual-luciferase reporter gene assay}

The conserved DNA binding sequence of rats STAT3 (TTCCGGGAA) were subcloned into pGL3 Basic plasmid (Promega, \#E1751) ${ }^{55}$. NRCMs were seeded at $5 \times 10^{4}$ cells per well into 96-well microplates, and were transiently co-transfected with the luciferase reporter (100 ng/well) and pRL-TK reporter constructs (Promega, E2241) at $20 \mathrm{ng} /$ well. The total content of transfected DNA was normalized by empty vector. After the indicated treatments, the luciferase activity was determined by the dual-luciferase reporter assay system (Promega, \#E1980) on a microplate reader (TECAN Infinite M1000).

\section{Statistical Analysis}

Graph Pad Prism 6.0 (Graph Pad software) or SPSS Version 21 was used for statistical analysis. Normality of the obtained data was assessed using a Shapiro-Wilk test. When normality was confirmed, statistical differences among groups were analyzed using either Student's t test (for two groups) or one (or two) -way analysis of variance (ANOVA, for more than two groups). Otherwise, the non-parametric test Kruskal-Wallis test followed by the Dunn's post-hot test was used to correct for multiple comparisons. In all cases, differences were considered statistically significant at a $P$ value less than 0.05 .

\section{Declarations}

\section{Acknowledgments}

This research was supported by grants from the National Natural Science Foundation of China (81803521, 81872860, 81673433), Local Innovative and Research Teams Project of Guangdong Pearl River Talents Program (2017BT01Y093), National Major Special Projects for the Creation and Manufacture of New Drugs (2019ZX09301104), National Engineering and Technology Research Center for New drug Druggability Evaluation (Seed Program of Guangdong Province, 2017B090903004), Foundation from Guangdong Traditional Medicine Bureau (20191060), Medical Scientific Research Foundation of Guangdong Province (A2018078), Fundamental Research Funds for the Central 
Universities (19ykpy131), and Special Program for Applied Science and Technology of Guangdong Province (2015B020232009).

\section{Author contributions}

J.L. and P.Q.L. conceived the project. J.L., Z.K.W., Y.H.H., J.J.W. and X.L.Z. designed and performed majority of the experiments and data analyses. S.W.X., Y.Q.H. and P.Q.L. provided scientific advice. D.P.S., P.X.W., Z.M.L. and M.Y.L. performed several in vitro experiments. J.L., Z.K.W. and P.Q.L. wrote the manuscript. All authors critically evaluated the manuscript.

\section{Competing interests}

The authors declare no competing interests.

\section{References}

1. A. Brown, J.B. Perry, M.E. Allen, H.N. Sabbah, B.L. Stauffer, S.R. Shaikh, J.G.F. Cleland, W.S. Colucci, J. Butler, A.A. Voors, S.D. Anker, B. Pitt, B. Pieske, G. Filippatos, S.J. Greene, M. Gheorghiade, Mitochondrial function as a therapeutic target in heart failure. Nat. Rev. Cardiol. 14, 238-250 (2017).

2. L. Roger, A.S. Go, D.M. Lloyd-Jones, E.J. Benjamin, J.D. Berry, W.B. Borden, D.M. Bravata, S. Dai, E.S. Ford, C.S. Fox, H.J. Fullerton, C. Gillespie, S.M. Hailpern, J.A. Heit, V.J. Howard, B.M. Kissela, S.J. Kittner, D.T. Lackland, J.H. Lichtman, L.D. Lisabeth, D.M. Makuc, G.M. Marcus, A. Marelli, D.B. Matchar, C.S. Moy, D. Mozaffarian, M.E. Mussolino, G. Nichol, N.P. Paynter, E.Z. Soliman, P.D. Sorlie, N. Sotoodehnia, T.N. Turan, S.S. Virani, N.D. Wong, D. Woo, M.B. Turner, C. Amer Heart Assoc Stat, S. Stroke Stat, Heart disease and stroke statistics-2012 update a report from the american heart association. Circulation 125, E2-E220 (2012).

3. Heineke, J.D. Molkentin, Regulation of cardiac hypertrophy by intracellular signalling pathways. Nat. Rev. Mol. Cell. Biol. 7, 589-600 (2006).

4. Kunisada, S. Negoro, E. Tone, M. Funamoto, T. Osugi, S. Yamada, M. Okabe, T. Kishimoto, K. Yamauchi-Takihara, Signal transducer and activator of transcription 3 in the heart transduces not only a hypertrophic signal but a protective signal against doxorubicin-induced cardiomyopathy. Proc. Natl. Acad. Sci. U. S. A. 97, 315-319 (2000).

5. Kovtun, N. Leneva, Y.S. Bykov, N. Ariotti, R.D. Teasdale, M. Schaffer, B.D. Engel, D.J. Owen, J.A.G. Briggs, B.M. Collins, Structure of the membrane-assembled retromer coat determined by cryo-electron tomography. Nature 561, 561-564 (2018).

6. Lucas, D.C. Gershlick, A. Vidaurrazaga, A.L. Rojas, J.S. Bonifacino, A. Hierro, Structural mechanism for cargo recognition by the retromer complex. Cell 167, 1623-1635.e14 (2016). 
7. S. Joyal, S. Nim, T. Zhu, N. Sitaras, J.C. Rivera, Z. Shao, P. Sapieha, D. Hamel, M. Sanchez, K. Zaniolo, M. St-Louis, J. Ouellette, M. Montoya-Zavala, A. Zabeida, E. Picard, P. Hardy, V. Bhosle, D.R. Varma, F. Gobeil, C. Beausejour, C. Boileau, W. Klein, M. Hollenberg, A. Ribeiro-da-Silva, G. Andelfinger, S. Chemtob, Subcellular localization of coagulation factor II receptor-like 1 in neurons governs angiogenesis. Nat. Med. 20, 1165-1173 (2014).

8. J. Cullen, H.C. Korswagen, Sorting nexins provide diversity for retromer-dependent trafficking events. Nat. Cell. Biol. 14, 29-37 (2011).

9. J. Cullen, Endosomal sorting and signalling: an emerging role for sorting nexins. Nat. Rev. Mol. Cell. Biol. 9, 574-582 (2008).

10. Zhou, Y. You, W. Shen, Y.Z. Zhu, J. Peng, H.T. Feng, Y. Wang, D. Li, W.W. Shao, C.X. Li, W.Z. Li, J. Xu, X. Shen, Deficiency of sorting nexin 10 prevents bone erosion in collagen-induced mouse arthritis through promoting NFATc1 degradation. Ann. Rheum. Dis. 75, 1211-1218 (2016).

11. Li, C.M. Li, D.S. Zhang, D. Shi, M. Qi, J. Feng, T.Y. Yuan, X.R. Xu, D.D. Liang, L. Xu, H. Zhang, Y. Liu, J.J. Chen, J.C. Ye, W.F. Jiang, Y.Y. Cui, Y.Y. Zhang, L.Y. Peng, Z.N.A. Zhou, Y.H. Chen, SNX13 reduction mediates heart failure through degradative sorting of apoptosis repressor with caspase recruitment domain. Nat. Commun. 5, 5177 (2014).

12. Okada, W. Zhang, C. Peterhoff, J.C. Hwang, R.A. Nixon, S.H. Ryu, T.W. Kim, Proteomic identification of sorting nexin 6 as a negative regulator of BACE1-mediated APP processing. FASEB J. 24, 2783-2794 (2010).

13. L. Zhang, K. Li, Y.G. Zhang, R. Lu, S.P. Wu, J.R. Tang, Y.L. Xia, J. Sun, Deletion of sorting nexin 27 suppresses proliferation in highly aggressive breast cancer MDA-MB-231 cells in vitro and in vivo. BMC Cancer 19, 555 (2019).

14. J. McGough, R.E.A. de Groot, A.P. Jellett, M.C. Betist, K.C. Varandas, C.M. Danson, K.J. Heesom, H.C. Korswagen, P.J. Cullen, SNX3-retromer requires an evolutionary conserved MON2:DOPEY2:ATP9A complex to mediate Wntless sorting and Wnt secretion. Nat. Commun. 9, 3737 (2018).

15. Feng, A.J. Streets, V. Nesin, U. Tran, H. Nie, M. Onopiuk, O. Wessely, L. Tsiokas, A.C.M. Ong, The sorting nexin 3 retromer pathway regulates the cell surface localization and activity of a Wntactivated polycystin channel complex. J. Am. Soc. Nephrol. 28, 2973-2984 (2017).

16. Cui, J.M. Carosi, Z. Yang, N. Ariotti, M.C. Kerr, R.G. Parton, T.J. Sargeant, R.D. Teasdale, Retromer has a selective function in cargo sorting via endosome transport carriers. J. Cell Biol. 218, 615-631 (2019).

17. Patel, C. Xu, S. Nagarajan, Z. Liu, W.O. Hemphill, R. Shi, V.N. Uversky, G.A. Caldwell, K.A. Caldwell, S.N. Witt, Alpha-synuclein inhibits Snx3-retromer-mediated retrograde recycling of iron transporters in S. cerevisiae and C. elegans models of Parkinson's disease. Hum. Mol. Genet. 27, 1514-1532 (2018).

18. Wang, Z. Li, Y. Tan, Q. Li, H. Yang, P. Wang, J. Lu, P. Liu, PARP1 interacts with STAT3 and retains active phosphorylated-STAT3 in nucleus during pathological myocardial hypertrophy. Mol. Cell Endocrinol. 474, 137-150 (2018). 
19. Zhang, X. Qu, B. Chen, M. Snyder, M. Wang, B. Li, Y. Tang, H. Chen, W. Zhu, L. Zhan, N. Yin, D. Li, L. Xie, Y. Liu, J.J. Zhang, X.Y. Fu, M. Rubart, L.S. Song, X.Y. Huang, W. Shou, Critical roles of STAT3 in beta-adrenergic functions in the heart. Circulation 133, 48-61 (2016).

20. X. Zhang, Z.S. Xu, H. Lin, M. Li, T. Xia, K. Cui, S.Y. Wang, Y. Li, H.B. Shu, Y.Y. Wang, TRIM27 mediates STAT3 activation at retromer-positive structures to promote colitis and colitis-associated carcinogenesis. Nat Commun 9, 3441 (2018).

21. Liu, K.M. McBride, N.C. Reich, STAT3 nuclear import is independent of tyrosine phosphorylation and mediated by importin-alpha 3. Proc. Natl. Acad. Sci. U. S. A. 102, 8150-8155 (2005).

22. C. Reich, L. Liu, Tracking STAT nuclear traffic. Nat. Rev. Immunol. 6, 602-612 (2006).

23. Ohara, Y. Fujita, K. Hata, M. Nakagawa, T. Yamashita, Axotomy induces axonogenesis in hippocampal neurons through STAT3. Cell Death Dis. 2, e175 (2011).

24. Ben-Yaakov, S.Y. Dagan, Y. Segal-Ruder, O. Shalem, D. Vuppalanchi, D.E. Willis, D. Yudin, I. Rishal, F. Rother, M. Bader, A. Blesch, Y. Pilpel, J.L. Twiss, M. Fainzilber, Axonal transcription factors signal retrogradely in lesioned peripheral nerve. EMBO J. 31, 1350-1363 (2012).

25. Di Liberto, V. Cavalli, Ready, STAT, go: transcription factors on the move. EMBO J. 31, 1331-1333 (2012).

26. Mukhopadhyay, M. Shah, F. Xu, K. Patel, R.M. Tuder, P.B. Sehgal, Cytoplasmic provenance of STAT3 and PY-STAT3 in the endolysosomal compartments in pulmonary arterial endothelial and smooth muscle cells: implications in pulmonary arterial hypertension. Am. J. Physiol. Lung Cell Mol. Physiol. 294, L449-L468 (2008).

27. H. Bild, J. Turkson, R. Jove, Cytoplasmic transport of Stat3 by receptor-mediated endocytosis. EMBO J. 21, 3255-3263 (2002).

28. L. German, B.M. Sauer, C.L. Howe, The STAT3 beacon: IL-6 recurrently activates STAT 3 from endosomal structures. Exp. Cell Res. 317, 1955-1969 (2011).

29. Kermorgant, P.J. Parker, Receptor trafficking controls weak signal delivery: a strategy used by c-Met for STAT3 nuclear accumulation. J. Cell Biol. 182, 855-863 (2008).

30. Li, J. Huang, J. Lu, Z. Guo, Z. Li, H. Gao, P. Wang, W. Luo, S. Cai, Y. Hu, K. Guo, L. Wang, Z. Li, M. Wang, X. Zhang, P. Liu, Sirtuin 1 represses PKC-zeta activity through regulating interplay of acetylation and phosphorylation in cardiac hypertrophy. Br. J. Pharmacol. 176, 416-435 (2019).

31. Hashimoto, G.E. Kim, R.S. Tunin, T. Adesiyun, S. Hsu, R. Nakagawa, G.S. Zhu, J.J. O'Brien, J.P. Hendrick, R.E. Davis, W. Yao, D. Beard, H.R. Hoxie, L.P. Wennogle, D.I. Lee, D.A. Kass, Acute enhancement of cardiac function by phosphodiesterase type 1 inhibition: translational study in the dog and rabbit. Circulation 138, 1974-1987 (2018).

32. G. Zhu, C. Wang, J.Z. Hu, R. Wan, J.H. Yu, J.Y. Xie, J.Y. Ma, L.J. Guo, J. Ge, Y.M. Qiu, L.F. Chen, H.L. Liu, X. Yan, X.X. Liu, J. Ye, W.F. He, Y. Shen, C. Wang, P.J. Mohler, K. Hong, Ankyrin-B Q1283H variant linked to arrhythmias via loss of local protein phosphatase 2A activity causes ryanodine receptor hyperphosphorylation. Circulation 138, 2682-2697 (2018). 
33. Li, Z. Lv, L. He, X. Huang, S. Zhang, H. Zhao, W. Pu, Y. Li, W. Yu, L. Zhang, X. Liu, K. Liu, J. Tang, X. Tian, Q.D. Wang, K.O. Lui, B. Zhou, Genetic tracing identifies early segregation of the cardiomyocyte and nonmyocyte lineages. Circ. Res. 125, 343-355 (2019).

34. Agah, P.A. Frenkel, B.A. French, L.H. Michael, P.A. Overbeek, M.D. Schneider, Gene recombination in postmitotic cells. Targeted expression of Cre recombinase provokes cardiac-restricted, site-specific rearrangement in adult ventricular muscle in vivo. J. Clin. Invest. 100, 169-179 (1997).

35. Kunisada, E. Tone, Y. Fujio, H. Matsui, K. Yamauchi-Takihara, T. Kishimoto, Activation of gp130 transduces hypertrophic signals via STAT3 in cardiac myocytes. Circulation 98, 346-352 (1998).

36. A. Frias, M.C. Rebsamen, C. Gerber-Wicht, U. Lang, Prostaglandin E2 activates Stat3 in neonatal rat ventricular cardiomyocytes: A role in cardiac hypertrophy. Cardiovasc. Res. 73, 57-65 (2007).

37. Chen, D. Garcia-Santos, Y. Ishikawa, A. Seguin, L. Li, K.H. Fegan, G.J. Hildick-Smith, D.I. Shah, J.D. Cooney, W. Chen, M.J. King, Y.Y. Yien, I.J. Schultz, H. Anderson, A.J. Dalton, M.L. Freedman, P.D. Kingsley, J. Palis, S.M. Hattangadi, H.F. Lodish, D.M. Ward, J. Kaplan, T. Maeda, P. Ponka, B.H. Paw, Snx3 regulates recycling of the transferrin receptor and iron assimilation. Cell Metab. 17, 343-352 (2013).

38. Temkin, B. Lauffer, S. Jager, P. Cimermancic, N.J. Krogan, M. von Zastrow, SNX27 mediates retromer tubule entry and endosome-to-plasma membrane trafficking of signalling receptors. Nat. Cell Biol. 13, 715-721 (2011).

39. F. Nothwehr, P. Bruinsma, L.A. Strawn, Distinct domains within Vps35p mediate the retrieval of two different cargo proteins from the yeast prevacuolar/endosomal compartment. Mol. Biol. Cell 10, 875890 (1999).

40. N. Seaman, J.M. McCaffery, S.D. Emr, A membrane coat complex essential for endosome-to-Golgi retrograde transport in yeast. J. Cell Biol. 142, 665-681 (1998).

41. Gallon, T. Clairfeuille, F. Steinberg, C. Mas, R. Ghai, R.B. Sessions, R.D. Teasdale, B.M. Collins, P.J. Cullen, A unique PDZ domain and arrestin-like fold interaction reveals mechanistic details of endocytic recycling by SNX27-retromer. Proc. Natl. Acad. Sci. U. S. A. 111, E3604-E3613 (2014).

42. Zhang, Y.H. Wu, T.Y. Belenkaya, X.H. Lin, SNX3 controls Wingless/Wnt secretion through regulating retromer-dependent recycling of Wntless. Cell Res. 21, 1677-1690 (2011).

43. J. Lorenowicz, M. Macurkova, T.C. Middelkoop, R. de Groot, M.C. Betist, H.C. Korswagen, Inhibition of late endosomal maturation restores Wnt secretion in Caenorhabditis elegans vps-29 retromer mutants. Cell Signal. 26, 19-31 (2014).

44. Harterink, F. Port, M.J. Lorenowicz, I.J. McGough, M. Silhankova, M.C. Betist, J.R.T. van Weering, R. van Heesbeen, T.C. Middelkoop, K. Basler, P.J. Cullen, H.C. Korswagen, A SNX3-dependent retromer pathway mediates retrograde transport of the Wnt sorting receptor Wntless and is required for Wnt secretion. Nat. Cell Biol. 13, 914-923 (2011).

45. I. Strochlic, B.C. Schmiedekamp, J. Lee, D.J. Katzmann, C.G. Burd, Opposing activities of the Snx3retromer complex and ESCRT proteins mediate regulated cargo sorting at a common endosome. Mol. Biol. Cell 19, 4694-4706 (2008). 
46. I. Strochlic, T.G. Setty, A. Sitaram, C.G. Burd, Grd19/Snx3p functions as a cargo-specic adapter for retromer-dependent endocytic recycling. J. Cell Biol. 177, 115-125 (2007).

47. R. Yin, T. Zhang, X.Y. Qu, Y.G. Zhang, R. Putatunda, X. Xiao, F. Li, W.D. Xiao, H.Q. Zhao, S. Dai, X.B. Qin, X.M. Mo, W.B. Young, K. Khalili, W.H. Hu, In vivo excision of HIV-1 provirus by saCas 9 and multiplex single-guide RNAs in animal models. Mol. Ther. 25, 1168-1186 (2017).

48. Tao, H. Yang, Q. Shi, Q. Fan, L. Wan, X. Lu, Targeted delivery to tumor-associated pericytes via an affibody with high affinity for PDGFRbeta enhances the in vivo antitumor effects of human TRAIL. Theranostics 7, 2261-2276 (2017).

49. Liu, J. Xu, Q. Ma, X. Zhang, Q. Yang, L. Wang, Y. Cao, Z. Xu, A. Tawfik, Y. Sun, N.L. Weintraub, D.J. Fulton, M. Hong, Z. Dong, L.E.H. Smith, R.B. Caldwell, A. Sodhi, Y. Huo, Glycolysis links reciprocal activation of myeloid cells and endothelial cells in the retinal angiogenic niche. Sci. Transl. Med. 12, eaay1371 (2020).

50. Lu, R.W. Zhang, H.Q. Hong, Z.L. Yang, D.P. Sun, S.Y. Sun, X.L. Guo, J.T. Ye, Z.M. Li, P.Q. Liu, The poly(ADP-ribosyl)ation of FoxO3 mediated by PARP1 participates in isoproterenol-induced cardiac hypertrophy. Biochim. Biophys. Acta 1863, 3027-3039 (2016).

51. Lu, D.P. Sun, Z.P. Liu, M. Li, H.Q. Hong, C. Liu, S. Gao, H. Li, Y. Cai, S.R. Chen, Z.M. Li, J.T. Ye, P.Q. Liu, SIRT6 suppresses isoproterenol-induced cardiac hypertrophy through activation of autophagy. Transl. Res. 172, 96-112 (2016).

52. E. de Araujo, G. Lamberti, L.A. Huber, Isolation of early and late endosomes by density gradient centrifugation. Cold Spring Harb. Protoc. 2015, 1013-1016 (2015).

53. Nakatsuka, M. Hayashi, A. Muroyama, M. Otsuka, S. Kozaki, H. Yamada, Y. Moriyama, D-aspartate is stored in secretory granules and released through a $\mathrm{Ca}^{2+}$-dependent pathway in a subset of rat pheochromocytoma PC12 cells. J Biol Chem 276, 26589-26596 (2001).

54. W. Luo, Y. Wang, H.W. Yang, C.M. Dai, H.L. Hong, J.Y. Li, Z.P. Liu, Z. Guo, X.Y. Chen, P. He, Z.Q. Li, F. Li, J.M. Jiang, P.Q. Liu, Z.M. Li, Heme oxygenase-1 ameliorates oxidative stress-induced endothelial senescence via regulating endothelial nitric oxide synthase activation and coupling. Aging. 10, 17221744 (2018).

55. M. Horvath, Z. Wen, J.E. Darnell, Jr., A STAT protein domain that determines DNA sequence recognition suggests a novel DNA-binding domain. Genes Dev. 9, 984-994 (1995).

\section{Figures}




\section{Figure 1}

a
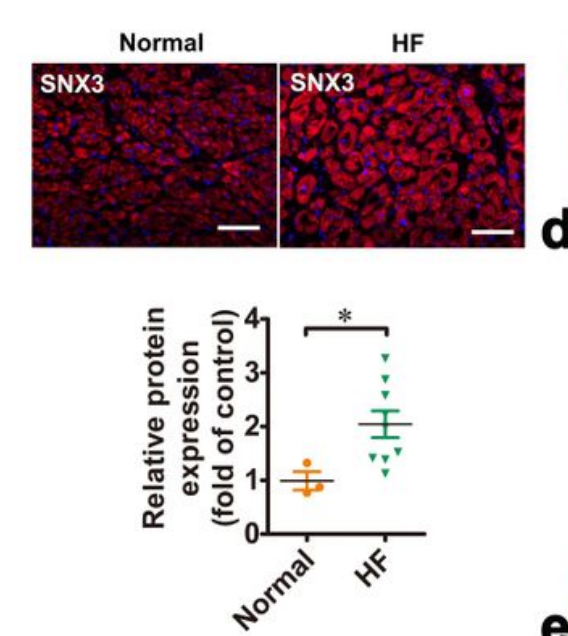

b
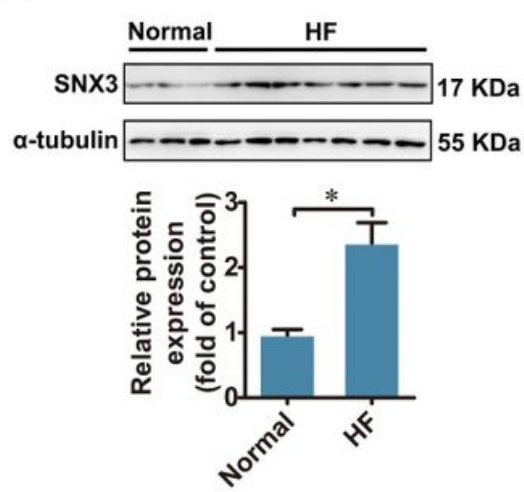

h
C
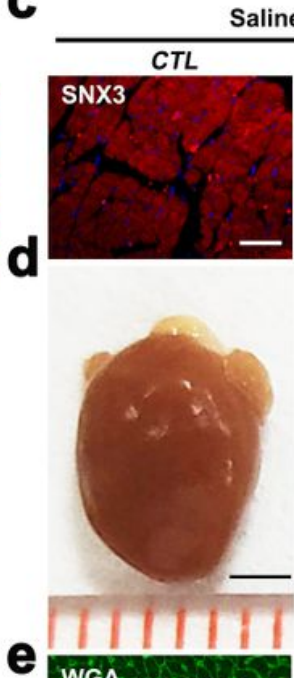

e wo

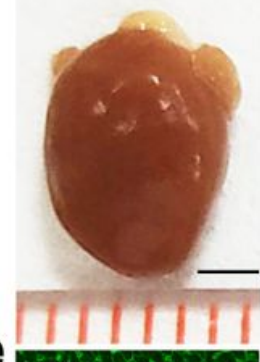

WGA
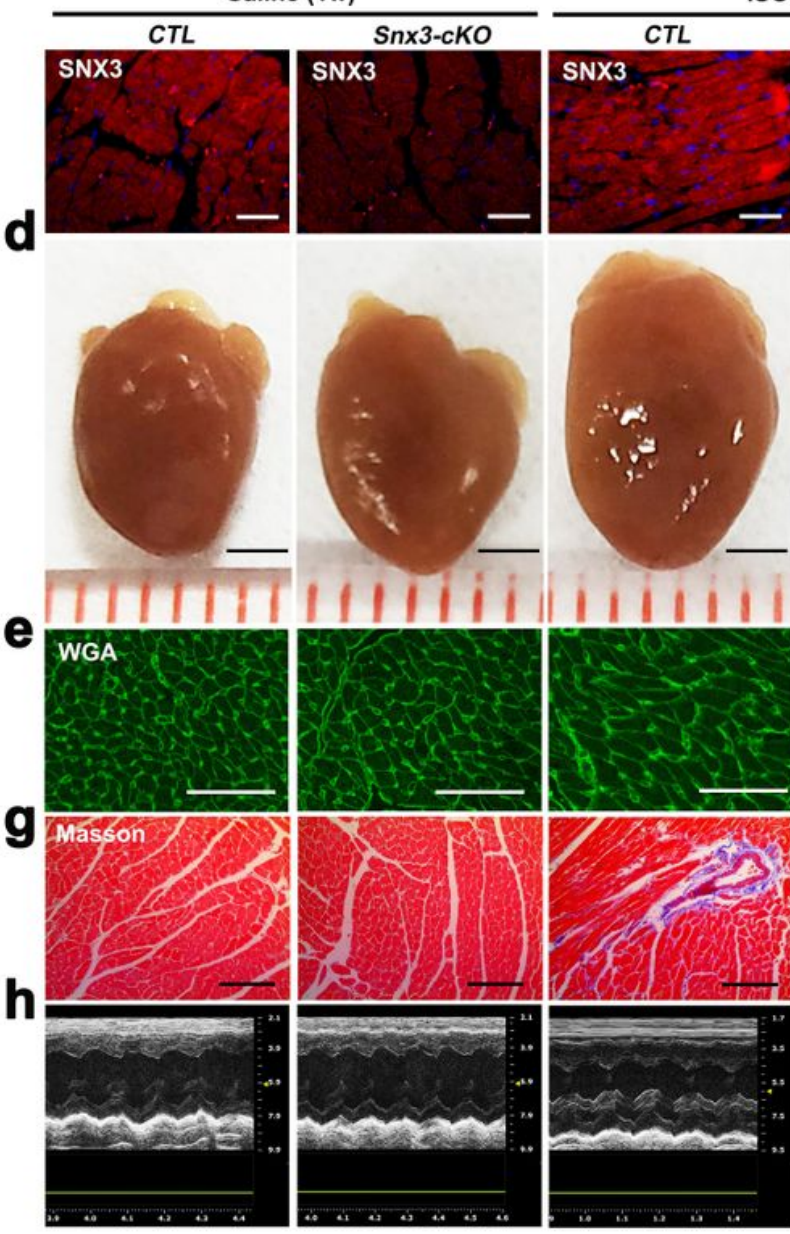

ISO (1w)

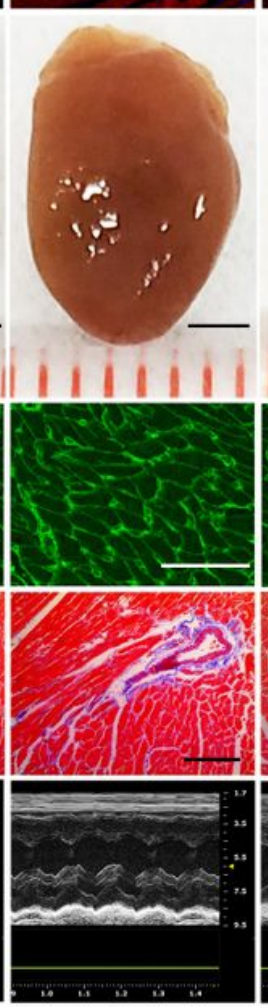

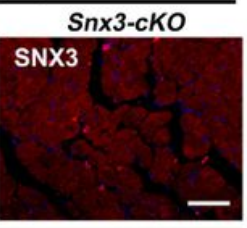
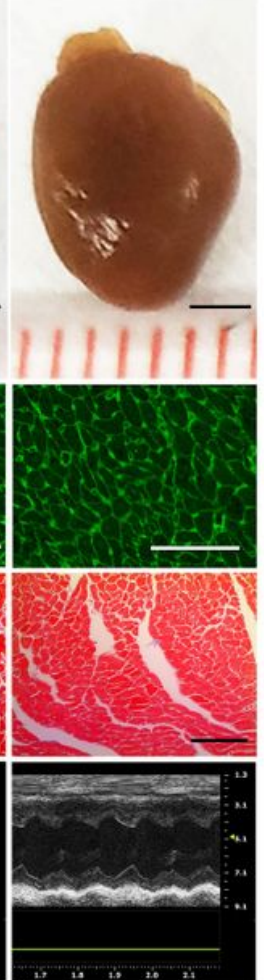

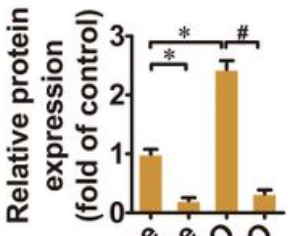

$\mathrm{n}^{2} \mathrm{~s}^{2} \mathrm{O}^{\mathrm{O}}$

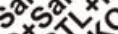

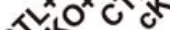

f

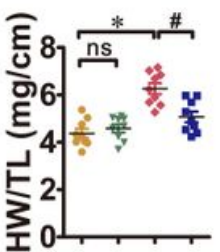
$\mathrm{CTL}+\cdot+\cdot$ Snx3-cKO - + - +

Saline $++\cdot \cdot$

ISO - $\cdot+$

- $C T L+$ Saline cKO+Saline CTL+ISO cKO+ISO

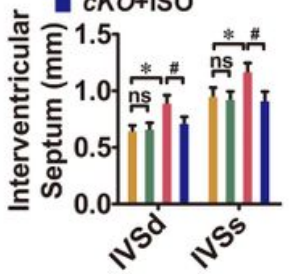

\section{Figure 1}

SNX3 expression was increased in failing human hearts, and cardiac-specific Snx3-cKO mice were protected from ISO-induced cardiac injury. $a$ and b A significant induction of SNX3 protein expression in end-stage failing human heart tissues ( $\mathrm{n}=9$ per HF, $\mathrm{n}=3$ per normal control), which was identified by IF assay (Scale bar: $200 \mu \mathrm{m}$ ) and western blot analysis. Male C57BL/6 Snx3-cKO mice and their respective CTL at 11 weeks were injected with ISO ( $3 \mathrm{mg} / \mathrm{kg} /$ day, s.c.) or an equal volume of sterile normal saline for one week. c The protein expression of SNX3 was measured bys IF assay (Scale bar: $100 \mu \mathrm{m}$ ). $\mathrm{d}$ and $\mathrm{e}$ Gross observation of heart morphology, WGA staining (Scale bar: $50 \mu \mathrm{m}$ ) -stained cross sections of heart tissues were shown. $f$ The HW/TL ratio was calculated. g Masson staining (Scale bar: $100 \mu \mathrm{m}$ ) -stained cross sections of heart tissues were shown. $\mathrm{h}$ The representative echocardiographic graphs were presented. $i$ The echocardiographic parameters IVS were measured. Representative images of five independent experiments were presented. The data were presented as the means $\pm S E M$. ${ }^{*} P<0.05$ vs. Normal or $C T L+$ Saline group, $\# P<0.05$ vs. $C T L+I S O$ group, $n=8-12$. Abbreviations: $C T L$, littermate negative control; $\mathrm{HF}$, heart failure; $\mathrm{HW} / \mathrm{TL}$, the heart weight to the tibia length ratio; IF, immunofluorescence; ISO, isoproterenol; IVS, interventricular septum; ns, no statistical difference; s.c., 
subcutaneously; Snx3-cKO, cardiac-specific Snx3 knockout; WGA, wheat germ agglutinin; 1 w, 1 week. See also Supplementary Figure S1-S3.

\section{Figure 2}

a

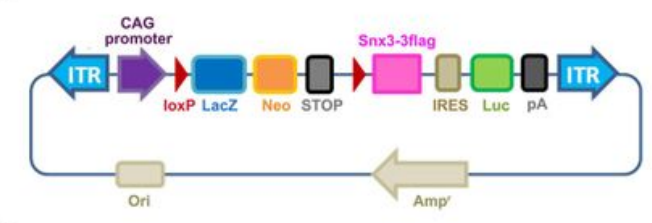

b

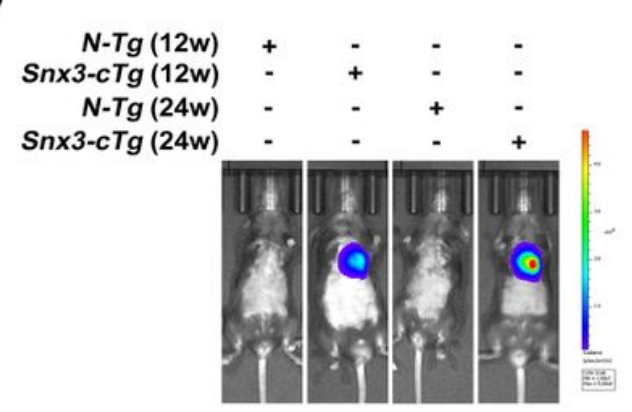

d

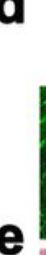

e
Saline (1w)

$N-T g(12 w)$ Snx3-cTg(12w) N-Tg(12w) Snx3-cTg $(12 w)$

$N-\operatorname{Tg}(24 \mathrm{w})$ Snx3-cTg $(24 \mathrm{w})$
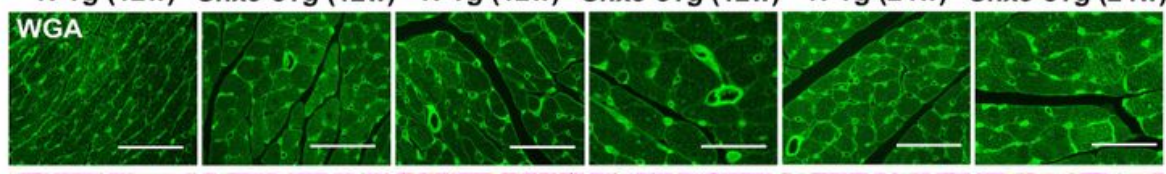

f
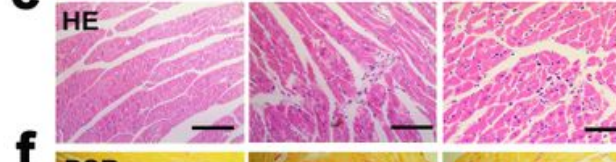

4.
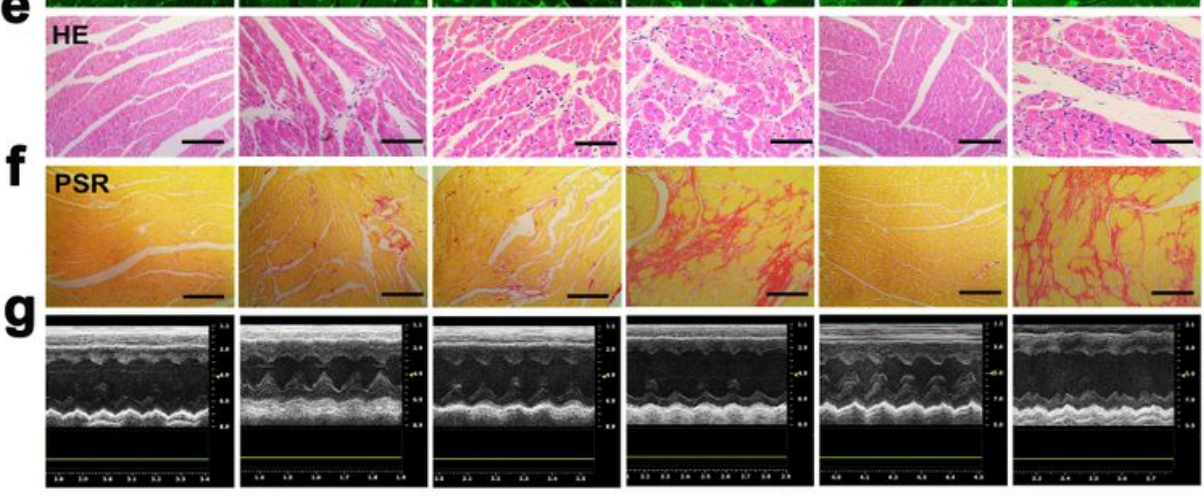

C

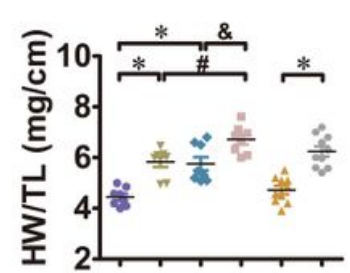

h

\section{i}
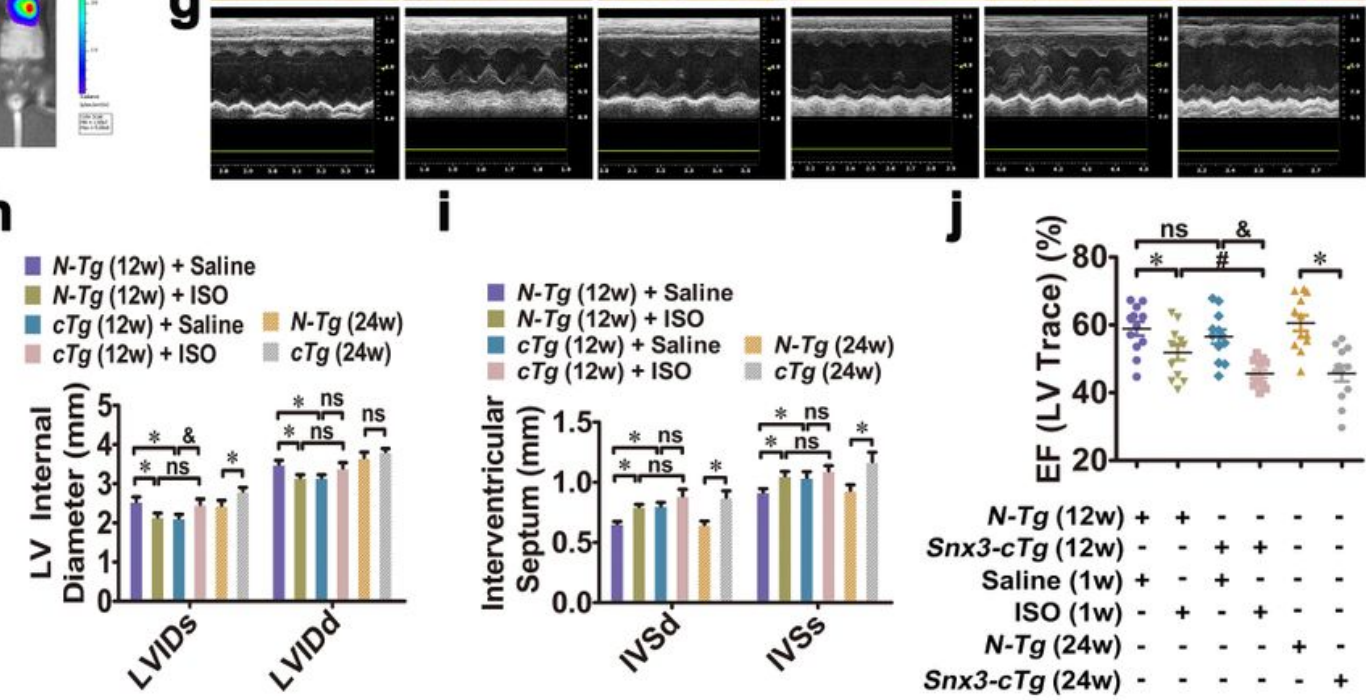

$N-\operatorname{Tg}(12 w)++\cdots \cdot-$

$\operatorname{Sn} \times 3-c T g(12 w)-\cdot++\cdot-$

Saline $(1 w)+\cdot+\cdot-\cdot$

ISO (1w) - + - + - -

$N-\operatorname{Tg}(24 w)-\cdot-++$

$\operatorname{Snx} 3-\operatorname{cTg}(24 \mathrm{w}) \cdot-\cdot \cdot+$

$N-\operatorname{Tg}(12 \mathrm{w})++\cdot-\cdot$

$\operatorname{Snx} 3-\operatorname{cTg}(12 w)-\cdot++\cdot \cdot$

Saline $(1 w)+\cdot+\cdot-\cdot$

ISO (1w) - + - + -

$N-T g(24 w)-\cdot-\cdot+\cdot$

$\operatorname{Snx} 3-\operatorname{cTg}(24 \mathrm{w}) \cdot-\cdot-\cdot+$

\section{Figure 2}

Cardiac-specific Snx3 transgene leaded to myocardial injury in mice. We investigated the changes of cardiac structure and function in Snx3-cTg mice at 12 and 24 weeks. Besides, Snx3-cTg mice (at 11 weeks) were injected with $3 \mathrm{mg} / \mathrm{kg} / \mathrm{d}$ ISO (s.c.) or an equal volume of sterile normal saline for one week. a The scheme of SnX3-cTg mice construction strategy was shown. b The protein level of SNX3 was detected by live imaging. $c$ The HW/TL ratio was calculated. d-f WGA staining (Scale bar: $50 \mu \mathrm{m}$ ), HE staining (Scale bar: $100 \mu \mathrm{m}$ ) and PSR staining (Scale bar: $100 \mu \mathrm{m}$ ) -stained cross sections of heart tissues were shown. $g$ The representative echocardiographic graphs were shown. $h-j$ The echocardiographic parameters (LVID, IVS and EF) were measured. Representative images of five independent experiments were presented. The data were shown as the means $\pm \mathrm{SEM}$. ${ }^{*}<<0.05 \mathrm{vs}$. N-Tg $(12 w)+$ Saline or N-Tg (24w) group, \#P< 0.05 vs. N-Tg (12w) + ISO group, \&P< 0.05 vs. Snx3-cTg (12w) + Saline group, $n=8-12$. Abbreviations: $E F$, ejection fraction; $H E$, hematoxylin-eosin; HW/TL, the heart weight to the tibia length ratio; ISO, isoproterenol; IVS, interventricular septum; LVID, left ventricular diameter; ns, no statistical difference; N-Tg, non-transgenic; PSR, picric sirius red; s.c., subcutaneously; 
Snx3-cTg, cardiac-specific Snx3 transgenic mice; WGA, wheat germ agglutinin; 1w, 1 week; 12 w, 12 weeks; 24w, 24 weeks. See also Supplementary Figure S4-S6.

\section{Figure 3}

a
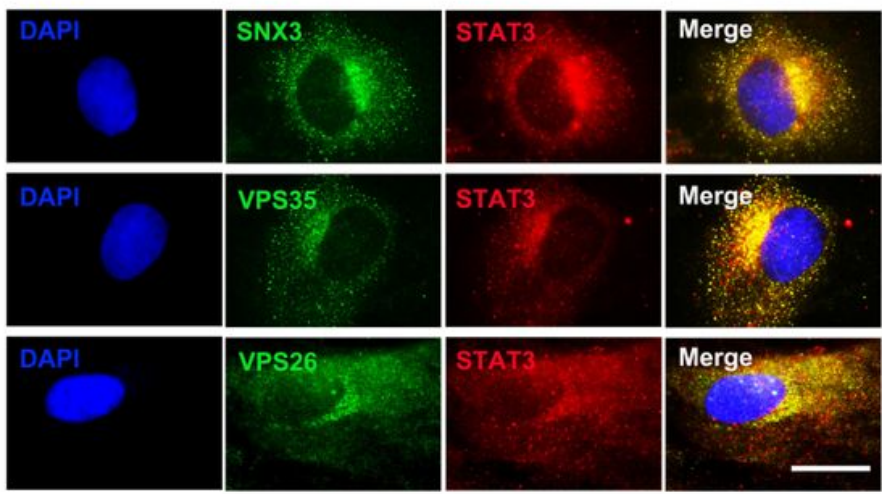

(1)
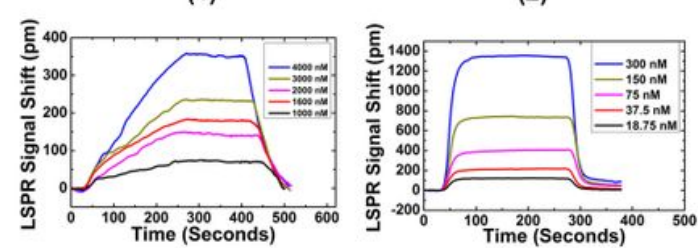

(2)
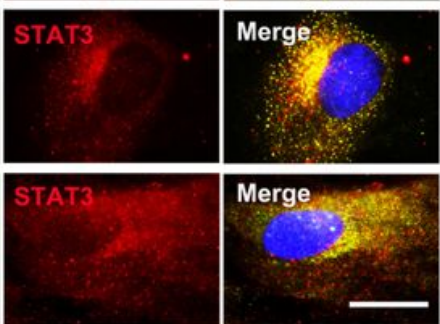

(3)
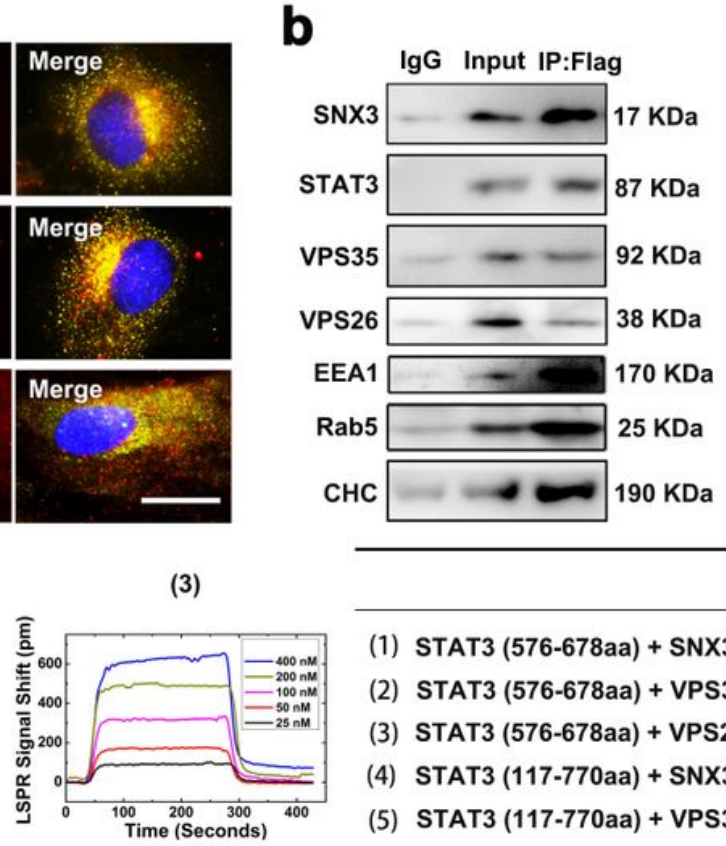

C

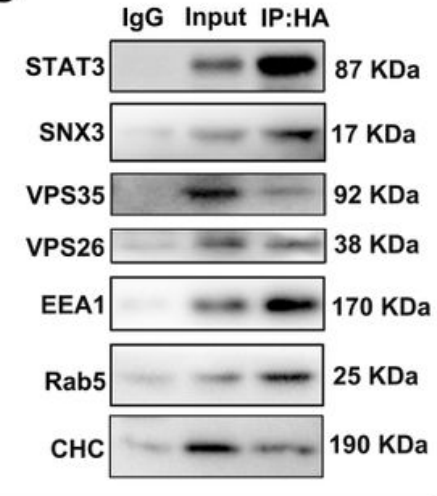

\begin{tabular}{lccc}
\hline & ka $\left(1 /\left(M^{\star} \mathrm{s}\right)\right)$ & kd (1s) & KD (M) \\
\hline (1) STAT3 (576-678aa) + SNX3 & $1.39 \mathrm{e} 3$ & $8.51 \mathrm{e}-5$ & $6.11 \mathrm{e}-8$ \\
(2) STAT3 (576-678aa) + VPS35 & $1.77 \mathrm{e} 5$ & $8.98 \mathrm{e}-3$ & $5.06 \mathrm{e}-8$ \\
(3) STAT3 (576-678aa) + VPS26 & $9.60 \mathrm{e} 4$ & $9.70 \mathrm{e}-3$ & $1.01 \mathrm{e}-7$ \\
(4) STAT3 (117-770aa) + SNX3 & $4.06 \mathrm{e} 3$ & $2.88 \mathrm{e}-3$ & $7.10 \mathrm{e}-7$ \\
(5) STAT3 (117-770aa) + VPS35 & $1.46 \mathrm{e} 4$ & $2.31 \mathrm{e}-4$ & $1.58 \mathrm{e}-8$ \\
(6) STAT3 (117-770aa) + VPS26 & $3.15 \mathrm{e} 4$ & $4.10 \mathrm{e}-4$ & $1.30 \mathrm{e}-8$ \\
\hline
\end{tabular}

e

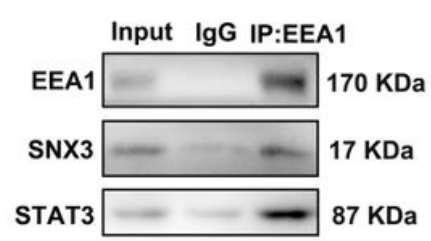

千

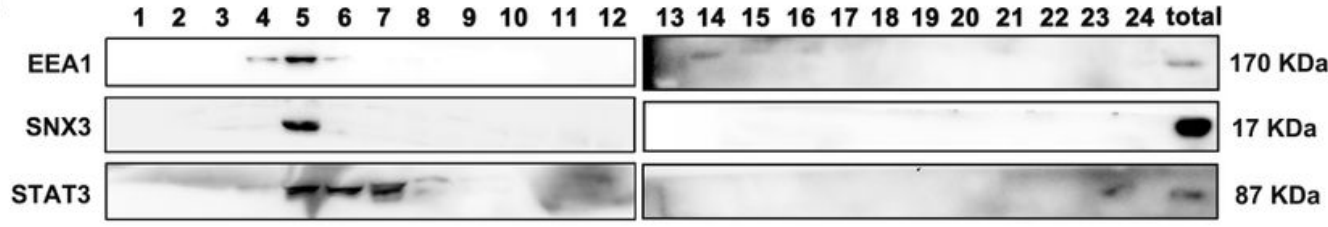

\section{Figure 3}

SNX3-retromer directly interacted with STAT3 at early endosomes in vivo and in vitro. a NRCMs were infected with Ad-STAT3 (HA-tagged) and were measured by IF staining using confocal microscopy (Scale bar: $25 \mu \mathrm{m})$. Representative images of five independent experiments were presented. NRCMs were infected with Ad-SNX3 (Flag-tagged) or Ad-STAT3 (HA-tagged), and were precipitated by anti-Flag (b) or anti-HA (c). d The binding curves of SNX3-retromer and truncated STAT3 protein (576-678aa) were measured by LSPR analysis, and the ka, kd and KD values for STAT3 (576-678aa, 117-720aa), SNX3, VPS35 and VPS26 were calculated by TraceDrawer ${ }^{\mathrm{TM}}$. e NRCMs were precipitated by anti-EEA1 (marker for early endosome) for SNX3 and STAT3 detection in co-IP assays. $f$ The early endosome fraction was purified from NRCMs using density gradient centrifugation, and detected by western blot. $\mathrm{n}=5$. Abbreviations: CHC, clathrin heavy chain; co-IP, co-immunoprecipitation; IP-MS, immunoprecipitationbased mass spectrometry; LSPR, localized surface plasmon resonance; NRCMs, neonatal rat cardiomyocytes. See also Supplementary Figure S7. 
Figure 4

a

Con

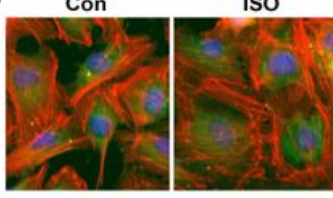

C

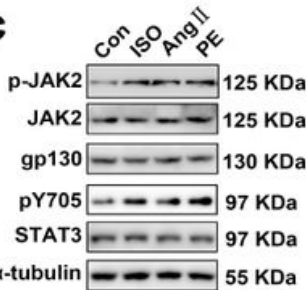

-tubulin $-2-55 \mathrm{KDa}$

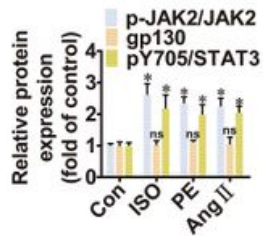

Ang II

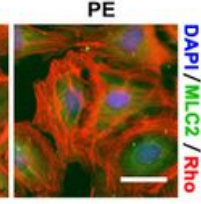

d

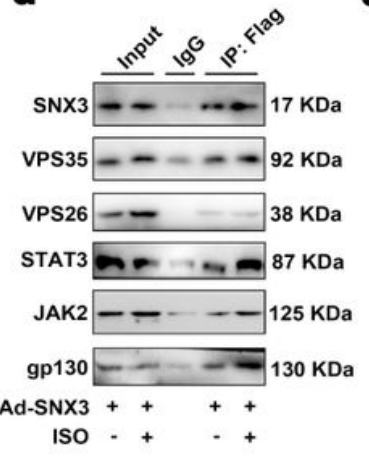

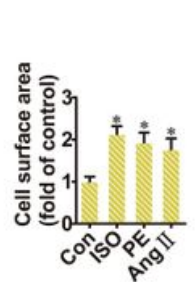

e

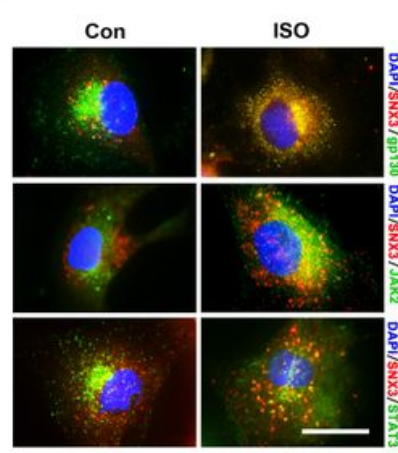

b

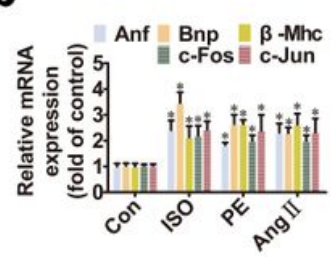

f

Input IgG IP: SNX3 JAK2 - $\ldots \ldots 125 \mathrm{KDa}$ gp130 - — — — $130 \mathrm{KDa}$ VPS 35 _ _ _ _.. $92 \mathrm{KDa}$ VPS26 - $20-\square \mathrm{KDa}$ STAT3 $-\ldots 8 \mathrm{KDa}$ Ad-NC + + - ++-
-+ ISO $-+\cdot+\cdots+\cdot$

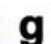

h

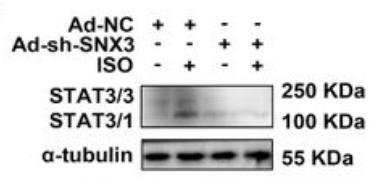
a-tubulin

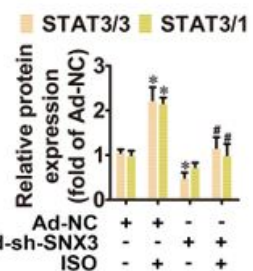

\section{Figure 4}

SNX3-retromer acted as a platform for STAT3 activation in NRCMs. NRCMs were treated with three hypertrophic stimulants, including ISO $(10 \mu \mathrm{mol} / \mathrm{L})$, Ang $\otimes(1 \mu \mathrm{mol} / \mathrm{L})$ and PE $(100 \mu \mathrm{mol} / \mathrm{L})$, respectively, for the times indicated. a The cell surface area was measured by staining with anti-MLC2 antibody (green) and rhodamine-phalloidin (red) (Scale bar: $25 \mu \mathrm{m}$ ). b The mRNA levels of Anf, Bnp, $\beta-M h c, c-F o s$ and c-Jun were determined by qPCR. c Western blot analysis was performed to detect the phosphorylated JAK2 (at tyrosine 1007 and 1008, p-JAK2) and STAT3 (at tyrosine 705, pY705), as well as the protein expression of JAK2, gp130 and STAT3. d NRCMs infected with Ad-SNX3 (Flag-tagged) were treated with ISO for $1 \mathrm{~h}$, and were precipitated by anti-Flag antibody, followed by co-IP assays. e The intracellular colocalization of SNX3 and gp130, JAK2, STAT3 in ISO-treated NRCMs was determined using confocal microscopy (Scale bar: $25 \mu \mathrm{m}$ ). NRCMs were infected with Ad-sh-SNX3 followed by incubation with ISO (10 $\mu \mathrm{mol} / \mathrm{L}$ for $1 \mathrm{~h})$, were precipitated by anti-SNX3 in co-IP assays (f), were detected the phosphorylated STAT3 (pY705), the protein expression of SNX3 and STAT3 (g), and were examined the protein expression of STAT3/3 homodimer or STAT3/1 heterodimer (h). Representative images of five independent experiments were presented. The data were shown as the means \pm SEM. ${ }^{\star} \mathrm{P}<0.05$ vs. control or Ad-NC

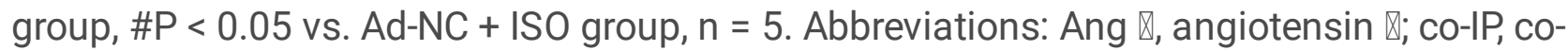
immunoprecipitation; IF, immunofluorescence; ISO, isoproterenol; MLC2, myosin light chain 2; NC, negative control; NRCMs, neonatal rat cardiomyocytes; PE, phenylephrine; qPCR, quantitative polymerase chain reaction; 1h, 1 hour. See also Supplementary Figure S8. 
Figure 5

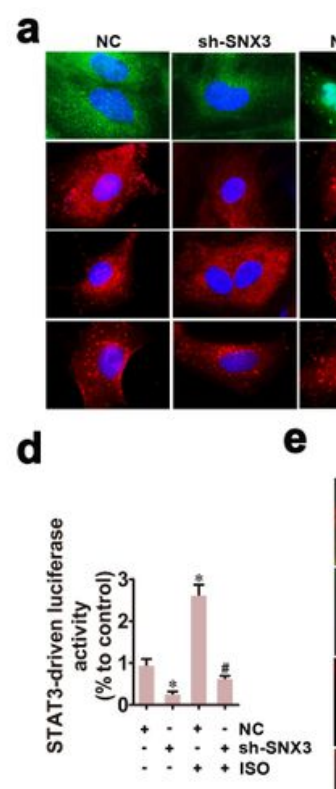

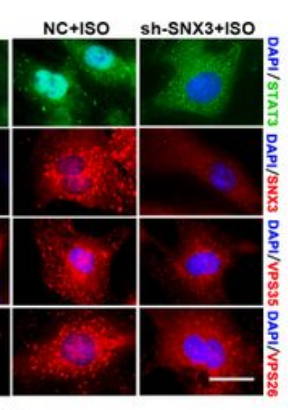

e

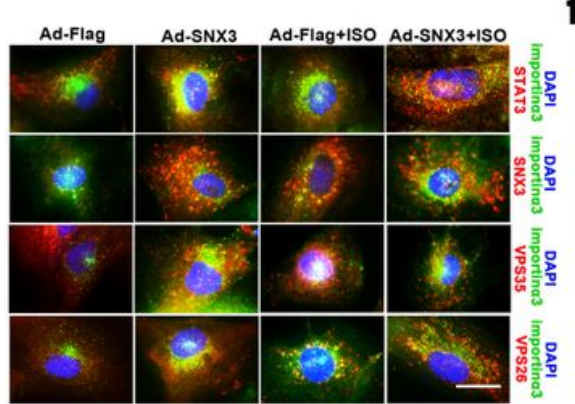

b
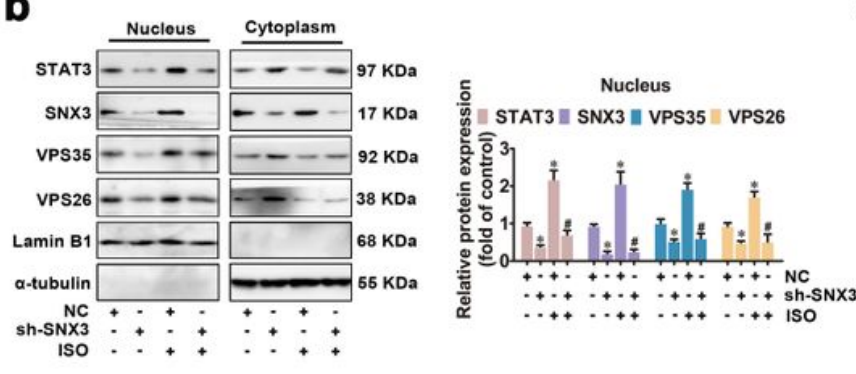

f

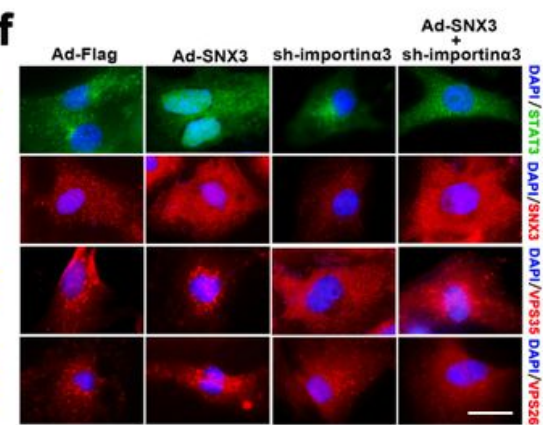

C
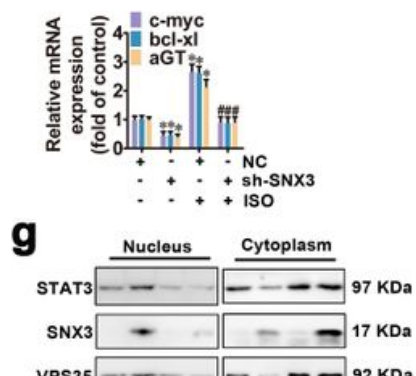

VPS35 _- - - $92 \mathrm{KDa}$

VPS26 - - - $38 \mathrm{KDa}$

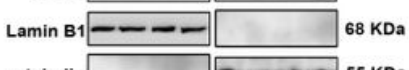

a-tubulin $\sim 65 \mathrm{KDa}$

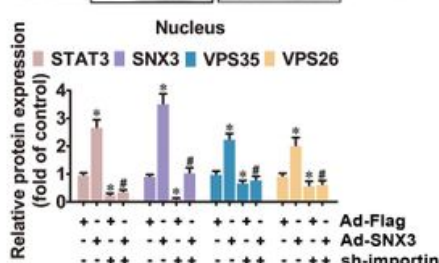

Figure 5

SNX3-retromer promoted the nuclear localization of STAT3 in NRCMs. NRCMs were infected with Ad-shSNX3 or Ad-SNX3 before ISO treatment for $1 \mathrm{~h}$. Besides, another group of NRCMs was infected with AdSNX3 followed by transfection with shRNAs of importin a3. (a, e and f) IF assay was performed to detect the subcellular distribution of STAT3, SNX3, VPS35 and VPS26. Representative images of five independent experiments were presented. ( $\mathrm{b}$ and $\mathrm{g}$ ) The nuclear and cytoplasmic proteins were extracted from NRCMs, and were detected by western blot analysis. The results were normalized to those of atubulin/Lamin B1. (c) Luciferase reporter gene assays showed the transcriptional activity of STAT3. (d) The mRNA levels of the target genes of STAT3 (c-myc, bcl-xl and aGT) was confirmed by qPCR. The data were shown as the means \pm SEM. *P $<0.05$ vs. NC or Ad-Flag group, \#P $<0.05$ vs. Ad-sh-SNX3 or Ad$\mathrm{SNX} 3$ group, $\mathrm{n}=5$. Abbreviations: IF, immunofluorescence; ISO, isoproterenol; NC, negative control; NRCMs, neonatal rat cardiomyocytes; qPCR, quantitative polymerase chain reaction. See also Supplementary Figure S9. 
Figure 6

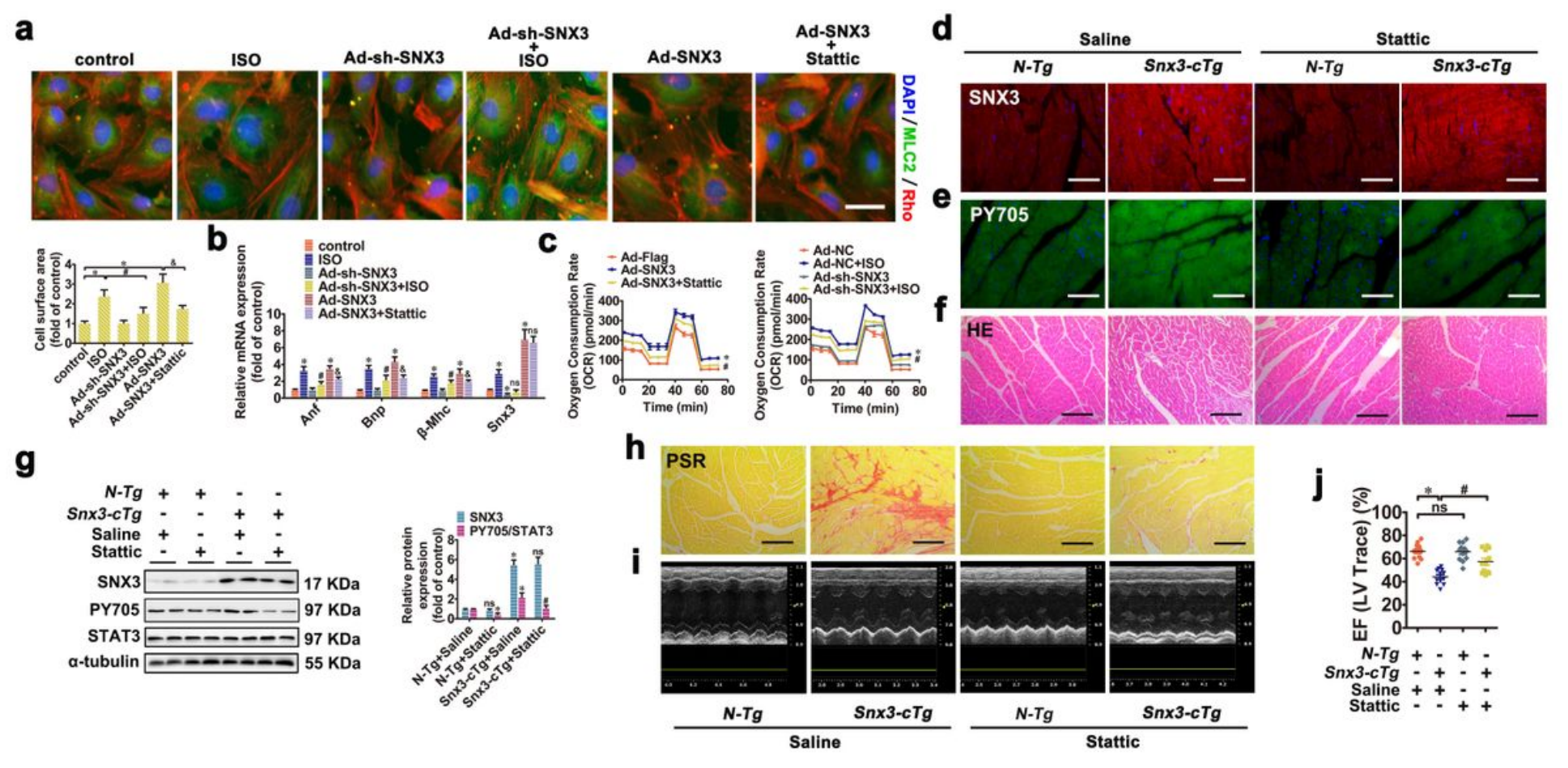

Figure 6

STAT3 was involved in SNX3-mediated cardiomyocyte hypertrophy in NRCMs and in Snx3-cTg mice. NRCMs were infected with Ad-sh-SNX3 before ISO treatment $(10 \mu \mathrm{mol} / \mathrm{L}, 1 \mathrm{~h})$, or were infected with AdSNX3 followed by treatment with stattic (a specific inhibitor of STAT3, $5 \mu \mathrm{mol} / \mathrm{L}, 24 \mathrm{~h}$ ). a The cell surface area was measured by staining with anti-MLC2 (green) and rhodamine-phalloidin (red) (Scale bar: 25 $\mu \mathrm{m})$. b The mRNA expression of Anf, Bnp, $\beta-M h c$ and Snx3 were measured by qPCR. c The OCR profile of NRCMs was measured by the high-throughput analyzer Seahorse XF96. Additionally, male Snx3-cTg mice (10-weeks-old) were administrated with stattic $(40 \mathrm{mg} / \mathrm{kg} / \mathrm{d}$, i.p.) or an equal volume of vehicle for 2 weeks. (d-f) The protein expression of SNX3 and the phosphorylated STAT3 (pY705) were measured by IF (Scale bar: $100 \mu \mathrm{m}$ ) and western blot analysis. (g and h) HE staining (Scale bar: $100 \mu \mathrm{m}$ ) and PSR staining (Scale bar: $100 \mu \mathrm{m}$ ) -stained cross sections of heart tissues were shown. $i$ The representative echocardiographic graphs were presented. $\mathrm{j}$ The echocardiographic parameter EF was measured.

Representative images of five independent experiments were presented. The data were presented as the means \pm SEM. $* \mathrm{P}<0.05$ vs. control, Ad-Flag, Ad-NC or N-Tg + Saline, group, \#P< 0.05 vs. ISO, Ad-SNX3 or Snx3-cTg + Saline group, \&P < 0.05 vs. Ad-sh-SNX3 or Ad-SNX3 group, $n=5$ or 8-12. Abbreviations: EF, ejection fraction; $\mathrm{HE}$, hematoxylin-eosin; IF, immunofluorescence; i.p., intraperitoneally; ISO, isoproterenol; MLC2, myosin light chain 2; NC, negative control; NRCMs, neonatal rat cardiomyocytes; ns, no statistical difference; N-Tg, non-transgenic; OCR, oxygen consumption; PSR, picric sirius red; qPCR, quantitative polymerase chain reaction; Snx3-cTg, cardiac-specific Snx3 transgenic; 1 h, 1 hour. See also Supplementary Figure S10 and S11.

\section{Supplementary Files}


This is a list of supplementary files associated with this preprint. Click to download.

- SupplementaryMaterial.docx 